\title{
Effects of bowing on perception of attractiveness
}

\author{
Takayuki Osugi • Jun I. Kawahara
}

Published online: 27 March 2015

(C) The Psychonomic Society, Inc. 2015

\begin{abstract}
Bowing is a greeting behavior. The present study examined the modulation effect of bowing on perception of attractiveness. In each trial, a portrait digitized from university yearbooks was presented on a computer screen. The portrait was mildly tilted toward participants to simulate a greeting bow (25-degree angle). Participants evaluated the subjective attractiveness of the face using a visual analog scale (0-100). The mean attractiveness judgment of the bowing portrait was significantly higher relative to that of the bending-backward or standing-still control conditions (Experiment 1). Additional control experiments revealed that alternative accounts relying on apparent spatial proximity and physical characteristics could not solely explain the effect of bowing (Experiment 2) and indicated that the effect was specific to objects perceived as faces (Experiment 3). Furthermore, observers' in-return bowing behavior did not reduce the bowing effect (Experiment 4), and bowing motion increased the ratings of subjective politeness and submissiveness (Experiment 5). Finally, tilting the 3D faces elicited the same effect from observers as did tilting the still photos (Experiment 6). These results suggest that a tilting motion of portraits (or images of face-like objects) mimicking bowing enhances perceived attractiveness, at least as measured in a culture familiar with greeting by bowing.
\end{abstract}

Keywords Facial attractiveness · Bowing motion · Greeting behavior $\cdot$ Pareidolia

T. Osugi

Department of Psychology, The University of Tokyo, Tokyo, Japan

J. I. Kawahara $(\triangle)$

Department of Psychology, Chukyo University, 101-2 Yagoto,

Showa, Nagoya 466-8666, Japan

e-mail: jkawa@lets.chukyo-u.ac.jp
Greeting is an act of human communication between individuals encountering each other and plays a critical role forming first impressions. For example, shaking hands, a prevalent greeting behavior used in Western cultures, reflects personality traits and is related to the hiring recommendations made by interviewers (Chaplin, Phillips, Brown, Clanton, \& Stein, 2000; Stewart, Dustin, Barrick, \& Darnold, 2008). Bowing is another type of greeting behavior commonly used in East Asia; it is used instead of shaking hands when people meet or depart. East Asian people generally believe that bowing contributes substantially to the establishment of a positive first impression; thus many (particularly Japanese) businesses introduce specific training programs for newly recruited employees that start with learning how to bow to customers and seniors. Given that shaking hands has an impact on impression formation in Western cultures (Chaplin et al., 2000; Stewart et al., 2008), we would expect bowing to have a similar effect in East Asian cultures. However, little is known about the relationship between bowing and impression formation.

In the present study, we focused on the effect of bowing on perceptions of attractiveness, because perceived attractiveness has been shown to affect a wide range of decisions involving social selection, such as mate choices (Buss \& Barns, 1986), hiring decisions (Cash \& Kilcullen, 1985), and judgments about the seriousness of crimes (Sigall \& Ostrove, 1975). Furthermore, people in some cultures (e.g., the Japanese) routinely bow as a way of behaving in a socially appropriate manner in interactions with other people. Thus, we speculated that bowing directed toward appropriate targets on appropriate occasions would increase positive reactions to the bower and increase his/her chances of being selected. Studies regarding facial attractiveness have traditionally focused on the physical characteristics of faces, such as symmetry (Møller \& Thornhill, 1998), averageness (Langlois \& Roggman 1990), and sexual dimorphism (Andersson, 1994). Recent studies 
have demonstrated that social cues, such as shifts in gaze (Mason, Tatkow, \& Macrae, 2005; Kampe, Frith, Dolan, \& Frith, 2001; Conway, Jones, DeBruine, \& Little, 2008), facial expressions (Reis et al., 1990), and head orientation (Kampe et al., 2001) affect the perception of physical attractiveness. For example, the attractiveness of faces can be jointly modulated by gaze direction (directed or averted) and facial expression (smiling or neutral). Specifically, Jones et al. (2006) demonstrated that perceived physical attractiveness increases when a face smiles at an observer, whereas it decreases when a face smiles away from an observer or the face presents a neutral expression. These findings suggest that the human perceptual system integrates a broad swath of information, ranging from physical properties related to beauty to social cues about the target's intentions, attitudes, and interpersonal relationships, when perceiving facial attractiveness to maximize the benefits of a potential encounter (Argyle \& Cook, 1976; Baron-Cohen, 1995; Emery, 2000). Given that the aforementioned channels of communication (i.e., gaze direction and facial expressions) and bowing are used in similar social greeting situations, it is reasonable to assume that bowing behavior also sends messages about intentions, attitudes, and interpersonal relationships and that this behavior affects the perceived attractiveness of the bower. However, no research has investigated the relationship between bowing and facial attractiveness. The present study examined this issue.

We tilted a portrait to simulate bowing to measure the effect of bowing on observers' perceptions of attractiveness, because this procedure enabled us to manipulate various types of motion. However, it should be noted that impressions of faces are influenced by their vertical orientation (Mignault \& Chaudhuri, 2003). Specifically, perceived dominance or submissiveness varies systematically as a function of the vertical head angle: faces tilted downward are perceived as more submissive than are upright and raised faces. Furthermore, a recent study demonstrated that a vertical orientation of the face also affects perceived attractiveness. Burke and Sulikowski (2010) systematically varied the vertical orientation of faces and observers rated the perceived masculinity or femininity and attractiveness of the faces. The results revealed that presenting female faces in upward angles decreased their perceived femininity and attractiveness, whereas presenting the same faces at downward angles increased those ratings. Furthermore, presenting male faces in upward angles increased their perceived masculinity, whereas presenting the same faces in downward angles decreased their perceived masculinity. These results indicate that the human perceptual system also integrates vertical head angle when determining facial impressions. However, previous studies did not manipulate the vertical motion of facial images; thus, the effect of dynamic changes in the angles of faces simulating bowing behavior on perceptions of attractiveness remains unclear.
The first five experiments in the present study examined whether simulated bowing affected perceptions of facial attractiveness by manipulating the vertical motion of facial images so that a two-dimensional facial image on a computer screen would appear to be bowing. We used the vertical motion of two-dimensional facial images to manipulate systematically the strength of the bowing motion. Because such illusory bowing motion simulated varying degrees of normal bowing behavior, it helped us to identify the features of bowing motion that increase perceived attractiveness. We assessed attractiveness under three conditions: bending forward, bending backward, and standing still (control; Fig. 1). Under the bending-forward condition, a portrait was tilted forward at about a 25-degree angle to mimic a greeting bow and was then returned to an upright position. Under the bending-backward condition, the portrait was tilted backward at the same angle and was then returned to an upright position (Fig. 2A). The portrait remained upright under the standing-still condition, which served as a control condition. In Experiment 1, observers viewed portraits under these conditions and rated the attractiveness of the faces. In Experiment 2, we examined the contributions of apparent spatial proximity and changes in the physical appearance of the images to the attractiveness ratings. In Experiment 3, we extended the generality of the effect of bowing using various types of stimulus sets, such as inverted faces, non-face meaningless shapes, and face-like objects. We examined potential explanations of the bowing effects in the Experiments 4 and 5. Furthermore, to strengthen our assumption that tilting a still photo would elicit the same effect in viewers as would tilting a three-dimensional face, Experiment 6 confirmed that a bowing motion would elicit a similar effect using movie clips of computer-generated female three-dimensional faces.

\section{Experiment 1}

In Experiment 1, we compared the scores for facial attractiveness under the bending forward, bending-backward, and standing-still conditions. If facial attractiveness were rated merely and solely based on the physical characteristics seen during the motion sequence, then the bending-forward and backward conditions would elicit comparable ratings. In contrast, if the social information involving bowing affected attractiveness judgments, then ratings under the bendingforward condition would be higher than would those under the standing-still condition. Additionally, we examined whether the results were specific to the bending-forward motion or were systematically affected as a function of the angle. If attractiveness ratings depended on the vertical angle, we would expect an increase in attractiveness under the bending-forward condition as well as a decrease in attractiveness under the bending-backward condition relative to the 


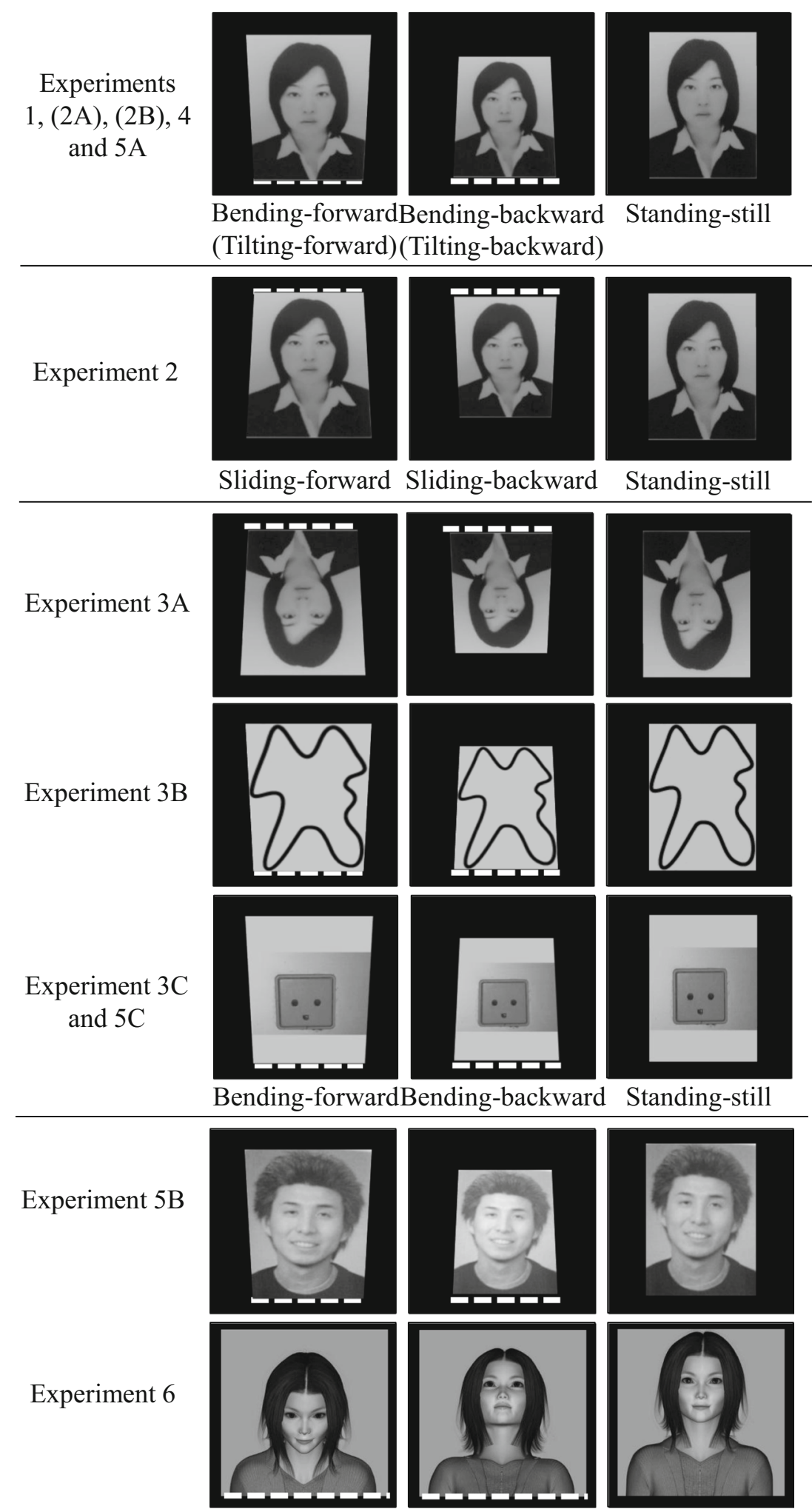

Bending-forwardBending-backward Standing-still

Fig. 1 Top row: Examples of the stimuli used. The face shown (presented with permission of the person) was not used in the actual experiments. The portraits were dynamically tilted 25 degrees from the upright position and then returned to the upright position under the bending-forward (left) and bending-backward (middle) conditions. The portraits remained upright under the standing-still condition (right). The segmented lines indicate the rotation axis. The second and the subsequent rows represent stimuli used in the following experiments 

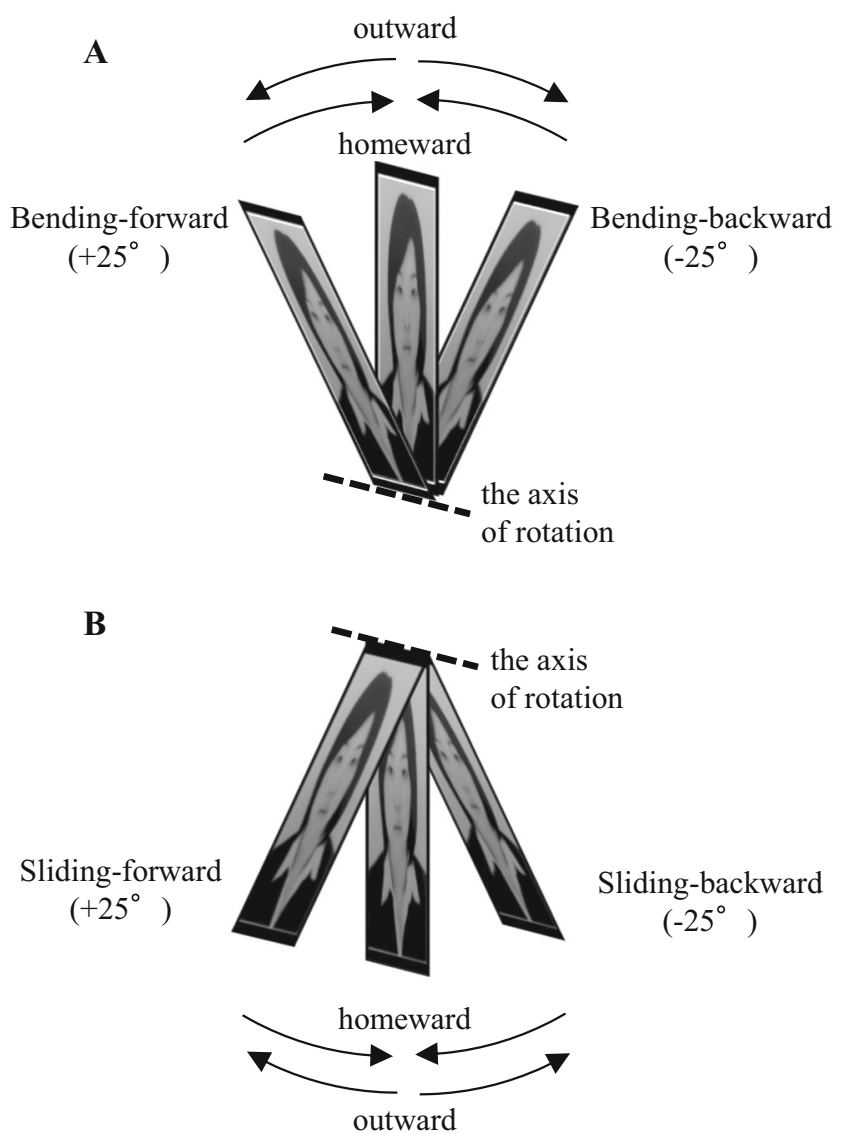

Fig. 2 Schematic diagram of the bowing motion. (A) The topside of the portrait was dynamically tilted forward or away around the lower side of the portrait at approximately 25 degrees for $500 \mathrm{~ms}$ (outward) and was returned to the upright position for the same duration (homeward). This type of motion was used in Experiments 1, 2A, 2B, 3B, 3C, 4, and 5. (B) The portraits moved with a bottom rotation axis in Experiments 2 and $3 \mathrm{~A}$

ratings under the standing-still condition because perceived dominance (submission) or masculinity (femininity) would systematically vary as a function of vertical head angle.

\section{Methods}

Participants Eighteen Japanese undergraduate students (mean age, 19.6 years, $\mathrm{SD}=1.0,8$ females) participated for pay. They were recruited from the Chukyo University subject pool for this and the following experiments. All reported normal or corrected-to-normal visual acuity and normal color vision.

Stimuli and Apparatus Stimuli were displayed on a liquidcrystal display (LL-193A-B, SHARP, $1280 \times 1024$ pixels) and controlled by a computer operating Microsoft Windows. The experimental procedure and rendering of the stimulus display were programmed using the open GL utility toolkit (GLUT) and $\mathrm{C}++$. The viewing distance was $75 \mathrm{~cm}$, and the monitor was placed on a table so that the bottom of the monitor was $140 \mathrm{~cm}$ above the floor.

Stimuli consisted of upright color portraits of 48 Japanese female faces with different clothing and hairstyles digitized from university yearbooks. Each portrait subtended $18.8 \mathrm{~cm}$ in height and $13.5 \mathrm{~cm}$ in width. All displayed a neutral expression or a faint smile. We used the same stimulus set of 1,932 female portraits used in the study by Sato and Kawahara (2014). This set of portraits was pre-rated by 31 male participants who did not participate in the present experiments to provide baseline attractiveness ratings. During the baseline measurements, a single image was presented at the center of a computer screen, and the rater evaluated the subjective physical attractiveness of the face using a visual analog scale $(0$ $100)$. We used portraits in the middle-range of attractiveness (37.0; range, 36.8-37.2).

Design and Procedure Participants rated the portraits under three conditions: bending-forward, bending-backward, and standing-still. The experiment consisted of 144 trials (48 under each condition, mixed randomly within a block). Each identity (facial stimulus) appeared three times and was assigned with equal frequency to the three conditions. The experiment lasted approximately $30 \mathrm{~min}$.

Each trial began with a portrait presented at the center of the screen for 1,000 ms; this was followed by a sequence of tilting motions under the bending-forward and bendingbackward conditions (Fig. 3). During the tilting-motion sequence, the topside of the portrait tilted toward the observer at approximately 25 degrees for $500 \mathrm{~ms}$ with the lower side as the rotation axis and then returned to the upright position for the same duration (Fig. 2A). The bending-backward condition was the same as the bending-forward condition except that the image tilted in the reverse direction. The portrait remained upright for 2,000 ms under the standing-still condition. Then, participants evaluated the subjective attractiveness of the person by clicking a point on a visual analog scale ranging from 0 (unattractive) to 100 (attractive) that appeared under the portrait. The participants were instructed to rate the attractiveness of the person according to their own criteria. We did not specify the kind of judgments participants were making (e.g., potential or short-term partner). Responses were selfpaced, and the next trial started after presentation of a random noise patch displayed for $1,000 \mathrm{~ms}$.

Results

Figure 4 shows the mean ratings for facial attractiveness under the bending-forward, bending-backward, and standing-still conditions. The ratings under the bending-forward condition were $20 \%$ higher than were those under the other conditions. An analysis of variance (ANOVA) on the ratings treating the motion condition as the within-subjects factor identified a 

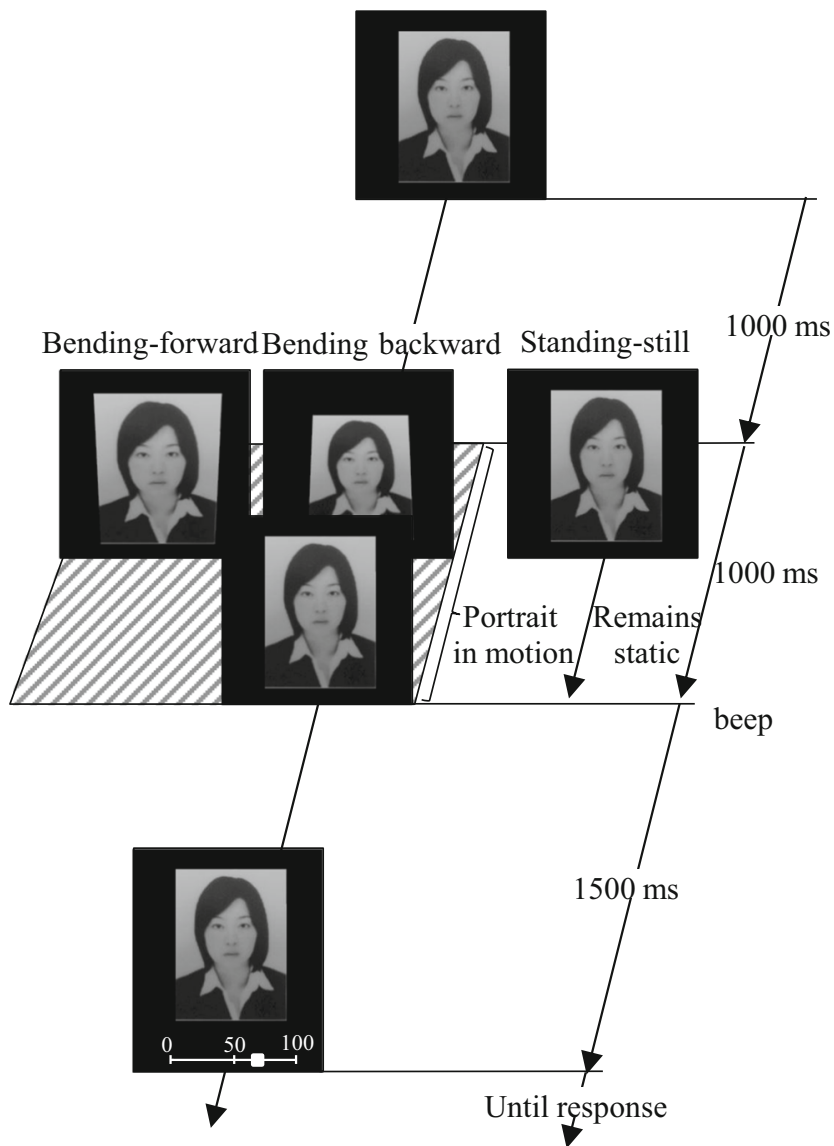

Fig. 3 Schematic diagram of the stimulus sequence. The portrait remained static for the first $1,000 \mathrm{~ms}$, followed by a $1,000-\mathrm{ms}$ period of tilting motion under the bending-forward and bending-backward conditions. The portrait remained static for the entire $3500 \mathrm{~ms}$ under the standing-still condition. Participants were prompted to enter a rating of attractiveness using a visual analog scale (0-100) that appeared under the portraits $1,500 \mathrm{~ms}$ after the end of the tilting-motion sequences

significant main effect, $F(2,34)=16.62, p<0.01, \eta_{p}{ }^{2}=0.49$. Multiple comparisons using the Bonferroni method (we used this method hereafter) indicated that the ratings under the bending-forward condition were significantly higher than were those under the bending-backward and standing-still conditions, $t s(17)>4.47, p s<0.01$, whereas no significant difference was observed between ratings under the bendingbackward and standing-still conditions, $t(17)=1.28, p=0.65$.

\section{Discussion}

The ratings for facial attractiveness under the bending-forward condition were higher than were those under the bendingbackward and standing-still conditions. This result indicates that the tilting motion of facial portraits mimicking bowing enhanced subjective attractiveness. Furthermore, the ratings under the bending-backward condition were comparable to those under the standing-still condition, suggesting that the results did not systematically change as a function of the
Experiment 1

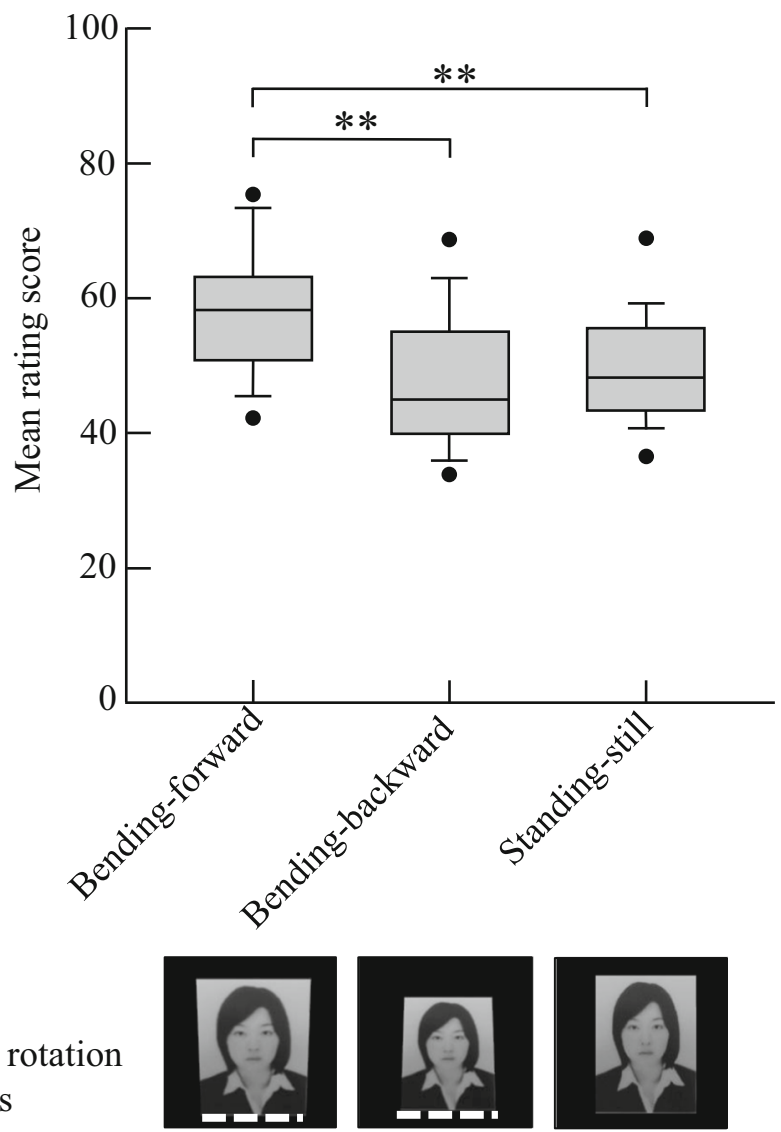

Fig. 4 Box plot for subjective attractiveness under the bending-forward, bending-backward, and standing-still conditions in Experiment 1. Each box shows the interquartile range, the horizontal line within the box shows the median, and the lines extending from the box to the highest and lowest values, excluding outliers. Outliers are marked separately. Black asterisks connected with the solid lines indicate significant differences between conditions $\left({ }^{* *} p<0.01,{ }^{*} p<0.05\right)$. The segmented lines in the pictures indicate the rotation axis

vertical head angle but were specific to the bending-forward condition. This result is consistent with our claim that the apparent bowing motion affects perception of facial attractiveness, reflecting the idea that Japanese generally believe that bowing contributes substantially to forming a positive first impression.

\section{Experiment 2}

Experiment 1 demonstrated that apparent bowing motion increases facial attractiveness, suggesting that perceiving the tilting motion of a portrait as a bow affects the perception of facial attractiveness. However, at least two alternative explanations should be considered. One involves the difference in the apparent spatial proximity between the stimulus and the observer, and the other involves the change in the physical 
characteristics of the faces. More specifically, differences in attractiveness ratings for the bending-forward, bending-backward, and standing-still conditions may have depended on differences in the apparent spatial proximity between stimulus and observer. Previous studies have demonstrated that reducing apparent spatial proximity (or the motivation latency to shorten the relative distance) is associated with positive ratings and that increasing apparent spatial proximity (or the motivation latency to increase the relative distance) is associated with negative ratings. For example, Cacioppo et al. (1993) demonstrated that approach-related action (i.e., pulling) improves the pleasantness of previously evaluated stimuli, whereas avoidance-related action (i.e., pushing) reduces pleasantness. Furthermore, observers responded to a positive stimulus more quickly when it moved toward them rather than away from them, whereas the reverse was true for negative stimuli (Markman \& Brendl, 2005).

Similarly, under the bending-forward condition in Experiment 1, observers may have rated attractiveness more positively when the stimulus was perceived as approaching them. Thus, it is possible that the enhanced effect of bowing observed in Experiment 1 was attributable to the decrease in relative distance. We conducted Experiment 2 by altering the axis of rotation from the bottom side to the topside of the portraits (Fig. 2B). Therefore, under the sliding-toward condition, the bottom side of the stimulus slid toward the observer; thus, this condition elicited the perception that the portrait was approaching. In contrast, sliding the bottom side away under the sliding-backward condition, elicited the perception that the portrait was retreating, representing avoidance. Therefore, if differences in apparent spatial proximity between the stimulus and observer were responsible for the effect found in Experiment 1, we would expect increased attractiveness under the sliding-toward condition and decreased attractiveness under the sliding-backward relative to the standing-still condition.

Second, we examined whether the change in the physical characteristics of faces was critical for the enhancement observed in Experiment 1. For example, a recent study demonstrated that presenting female faces at downward angles increases their perceived femininity, because these faces appear to have a larger forehead, larger eyes, and a smaller chin compared with those at upward angles (Burke \& Sulikowski, 2010). Similarly, a bowing motion might enhance their perceived attractiveness, because presenting faces in a downward angle might increase perceived femininity. If this were the case, the ratings under the slidingbackward condition would be higher than would be those under the sliding-toward and standing-still conditions, because the change in the apparent physical characteristics of faces under the sliding-backward condition would be the same as those in the bowing display used in Experiment 1 (the bending-forward condition).
Methods

The stimuli, apparatus, and procedures were the same as those in Experiment 1, except that the axes of rotation were altered from the bottom to the top of the portraits. That is, under the sliding-toward and sliding-backward conditions, the bottom side of the portrait slid toward (Fig. 1B, left column) and backward (Fig. 1B, middle column) around the topside, respectively. Fifteen undergraduate students (mean age, 20.0 years, $\mathrm{SD}=1.5,11$ females) participated for pay. All reported normal or corrected-to-normal visual acuity and normal color vision.

\section{Results and Discussion}

Figure 5 shows the mean ratings under the bending-forward, bending-backward, and standing-still conditions. ANOVA of

\section{Experiment 2}

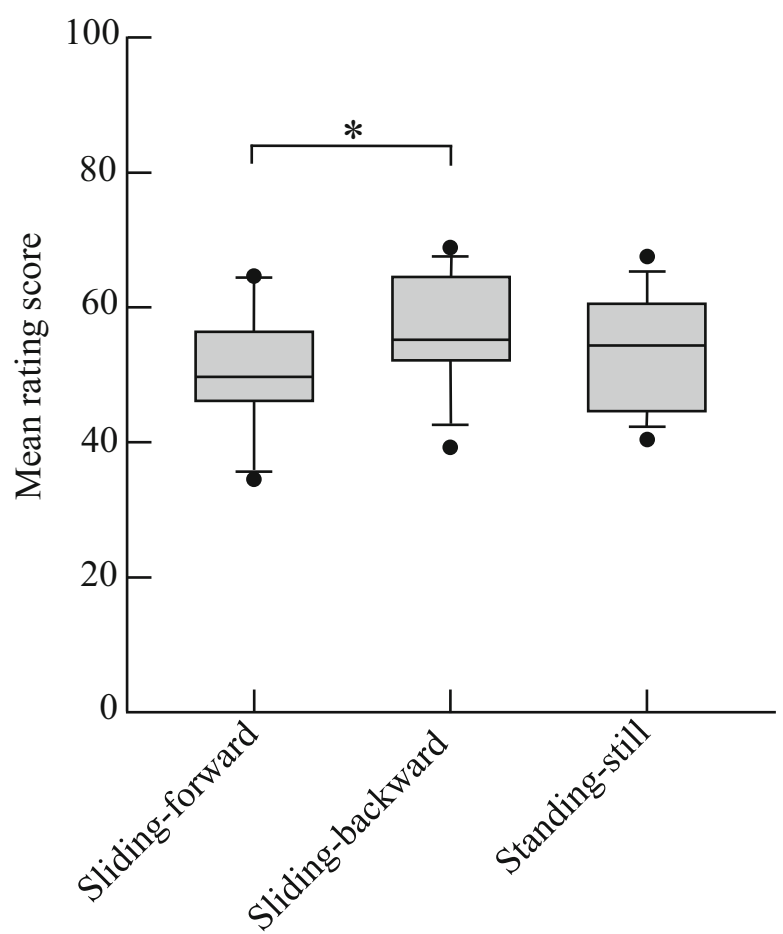

the rotation axis
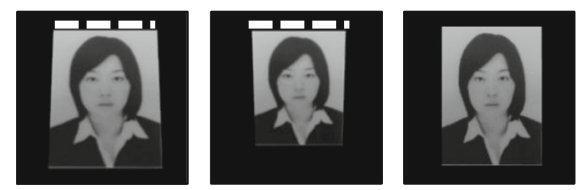

Fig. 5 Box plot for subjective attractiveness under the sliding-forward, sliding-backward, and standing-still conditions in Experiment 2. Each box shows the interquartile range, the horizontal line within the box shows the median, and the lines extending from the box to the highest and lowest values, excluding outliers. Outliers are marked separately. Black asterisks connected with the solid lines indicate significant differences between conditions $\left({ }^{* *} p<0.01,{ }^{*} p<0.05\right)$. The segmented lines in the pictures indicate the rotation axis 
the ratings identified a significant main effect, $F(2,28)=7.08$, $p<0.01, \eta_{p}{ }^{2}=0.34$. Multiple comparisons revealed that ratings under the sliding-backward condition were significantly higher than were those under the sliding-toward condition, $t(14)=2.79, p<0.05$. More importantly, no differences were found between the ratings under the standing-still and the other two conditions (i.e., the sliding-toward and backward conditions), $t s(14)<2.37, p s>0.08$.

The results revealed that the ratings under the slidingbackward condition were significantly higher than those under the sliding-toward condition. This result was inconsistent with alternative accounts relying on apparent spatial proximity, although physical deformations (e.g., larger forehead, smaller chin, or more widely spaced eyes) may contribute to increasing the attractiveness of a face. This pattern of results was consistent with the finding that female faces presented at downward angles appear to have larger foreheads, larger eyes, and a smaller chin compared with those presented at upward angles (Burke \& Sulikowski, 2010), resulting in increased perceived attractiveness.

However, the present result indicates that such deformation alone cannot explain the relatively large effect of bowing observed in Experiment 1. If the deformation of the physical characteristics of faces were critical to the enhancement of attractiveness ratings observed in Experiment 1, the ratings under the sliding-backward condition should have been higher than those under the standing-still condition, because the eventual degree of deformation under the sliding-backward condition was identical to that in the bowing display used in Experiment 1 (the bending-forward condition). In fact, no significant differences were found between the standing-still and the other two conditions. Thus, deformation of physical characteristics cannot be the sole contributor to the enhancement of attractiveness observed in Experiment 1. Alternatively, the major effect observed in Experiment 1 may mean that the tilting motion of the facial portraits, designed to simulate bowing, enhances perceived attractiveness. According to this view, the tilting motion is associated with socially desirable behaviors and therefore enhances physical attractiveness. Such a combined effect of the physical characteristics and the bowing motion may have induced the enhancement observed in Experiment 1. We further examined alternative explanations in Experiments 2A and 2B.

\section{Experiment 2A}

Experiment 2 demonstrated that changes in apparent distance did not increase facial attractiveness relative to the standingstill condition. However, the ratings under the sliding-toward and backward conditions significantly differed; thus, the results of Experiment 2 are consistent with the view that physical characteristics made some contribution to the results.
Indeed, one could argue that facial deformation, such as the head being larger when the topside of the portrait was bent (Experiment 1) than when the bottom side of the portrait was slid (Experiment 2), contributed to the results. This account posits that the effect of bowing on perception of attractiveness simply reflects the increased physical femininity that results from the change in viewing direction. To exclude this possibility, we used a display in which the topside of the portrait was tilted forward or backward to 25 degrees and maintained in this angle throughout each trial, and we examined the effect of the mere physical characteristics of the bowing face. Importantly, the physical characteristics of the face used in this experiment were the same as those of the face used when the portrait was subjected to the maximum tilt in Experiment 1. Thus, if the physical characteristics of the bowing face contributed to the induction of higher attractiveness ratings, the perceived attractiveness of the tilting-forward face would be higher than would be those of the standing-still and tiltingbackward faces.

\section{Methods}

Sixteen undergraduate male students (mean age, 19.2 years, $\mathrm{SD}=0.7,11$ females) participated for pay. All reported normal or corrected-to-normal visual acuity and normal color vision. The stimuli, apparatus, and procedures were the same as those in used Experiment 1 except that no motion was involved, the portraits were tilted forward or backward 25 degrees from the beginning of a trial, and the tilt was maintained during each trial under the tilting-toward and backward conditions, respectively.

\section{Results and Discussion}

Figure 6 shows the mean ratings under the tilting-toward, tilting-backward, and standing-still conditions. An ANOVA identified a significant main effect, $F(2,30)=14.95, p<$ $0.01, \eta_{p}{ }^{2}=0.50$. Multiple comparisons indicated that the ratings under the tilting-backward condition were significantly lower than were those under the tilting-toward and standingstill conditions, $t s(15)>4.07, p$ s $<0.01$. This result is consistent with the finding reported by Burke and Sulikowski (2010), in which the vertical orientation of the face affected the perception of attractiveness. However, no differences in ratings were found between the tilting-toward and standingstill conditions, $t(15)=1.43, p=0.52$, indicating that the effect of bowing cannot be attributed simply to the physical characteristics related to tilting the face toward an observer. That is, increasing the femininity did not contribute to the effect of bowing on perception of attractiveness under the present circumstances. Rather, we suggest that this effect reflects a preference for the tilting motion of those portraits that simulate bowing. 
Experiment 2A

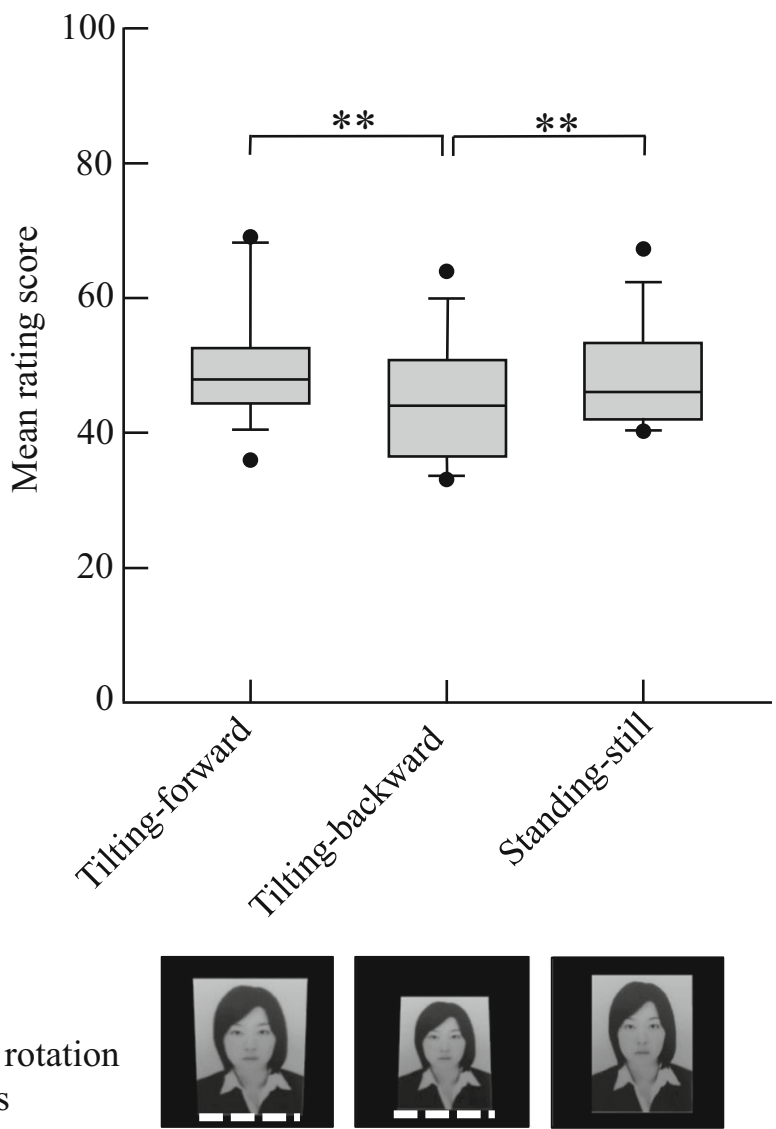

Fig. 6 Box plot for subjective attractiveness under the tilting-forward, tilting-backward, and standing-still conditions in Experiment 2A. Each box shows the interquartile range, the horizontal line within the box shows the median, and the lines extending from the box to the highest and lowest values, excluding outliers. Outliers are marked separately. Black asterisks connected with the solid lines indicate significant differences between conditions $\left({ }^{* *} p<0.01,{ }^{*} p<0.05\right)$. The segmented lines in the pictures indicate the rotation axis

\section{Experiment 2B}

Experiments 1 and 2 demonstrated that the effect of bowing on the perception of attractiveness reflected not only the increased physical femininity that resulted from the change in viewing direction but also the enhanced perceived attractiveness elicited by the tilting motion of facial portraits mimicking bowing. However, a bowing motion may exaggerate a facial deformation (e.g., making the head appear larger when the topside of the portrait looks larger than that of a static portrait) via mechanisms, such as representational momentum (Freyd \& Finke, 1984). Furthermore, it also is possible that the bowing motion is associated with a more attractive tilt of the portrait (e.g., a range of approximately 15 degrees) than is the maximum tilt tested in Experiment 2A, because the bowing motion involves a range of different perspectives. Thus, it is still possible that mere physical characteristics contributed to the results. To exclude these possibilities, the bending-forward condition was compared with the bending-backward and standing-still (control) conditions when participants were explicitly instructed to notice that the portrait was merely tilted toward or backward. If the effect of bowing reflected the increased physical femininity that results from the change in viewing direction, it should have been preserved under this condition.

Methods

Sixteen undergraduate male students (mean age, 18.6 years, $\mathrm{SD}=0.9,14$ females) participated for pay. All reported normal or corrected-to-normal visual acuity and normal color vision. The stimuli, apparatus, and procedures were the same as those used in Experiment 1, except that participants were explicitly instructed to notice that the portrait was merely tilted forward or backward.

Results and Discussion

Figure 7 shows the mean ratings under the bending-forward, bending-backward, and standing-still conditions. An ANOVA revealed no significant main effects, $F(2,30)=1.79, p=0.19$, $\eta_{p}{ }^{2}=0.01$. This result indicates that the effect of bowing could not be attributed simply to the physical characteristics induced by a bowing motion. That is, a bowing motion may not affect attractiveness ratings when participants do not see the bending-forward motion as a bow, suggesting that this effect reflects a preference for the tilting motion of those portraits when they simulate bowing. Furthermore, this result ruled out the alternative explanations that the bowing effect may be due to the facial deformation or the different perspective on the face.

Experiment 2 demonstrated that when participants did not see the tilted face as a bow, the attractiveness ratings of these faces did not increase relative to those of the upright static faces. This finding suggests that the increased attractiveness ratings were attributable primarily to the bowing motion. Although physical characteristics may have contributed to the present results, their effect seems small relative to that of bowing.

\section{Experiment 3}

Experiments 1 and 2 demonstrated that the bowing motion increased the perceived attractiveness of the faces. What remained unclear was the generality of the benefits related to bowing. In other words, it was unclear whether the effect was specific to objects perceived as faces. To examine this issue, we used three types of stimulus sets: inverted faces (Experiment $3 \mathrm{~A}$ ), non-face meaningless line drawings 
Experiment 2B

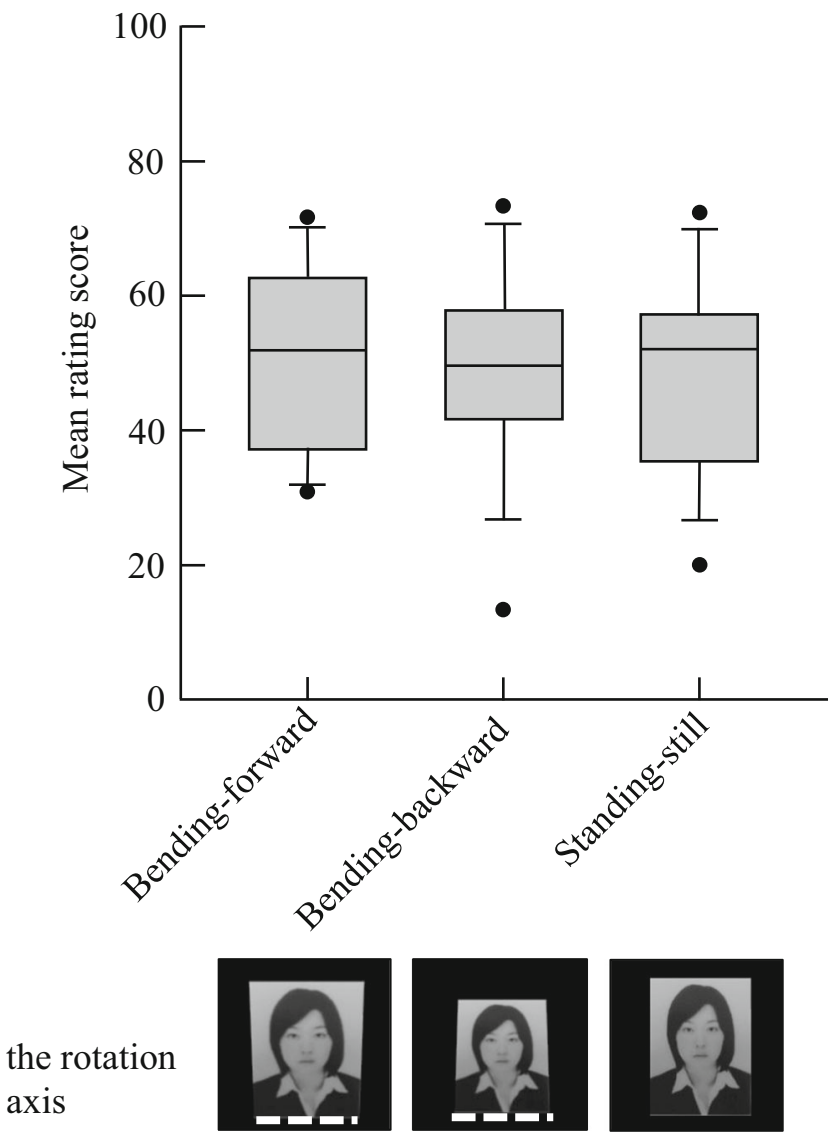

Fig. 7 Box plot for subjective attractiveness under the bending-forward, bending-backward, and standing-still conditions in Experiment 2B. Each box shows the interquartile range, the horizontal line within the box shows the median, and the lines extending from the box to the highest and lowest values, excluding outliers. Outliers are marked separately. The segmented lines in the pictures indicate the rotation axis

(Experiment 3B), and face-like objects, such as a picture of an electric outlet (Experiment 3C). We chose these stimuli for the following reasons. The adoption of inverted facial images allowed us to investigate the face-specific process because the human visual system is specialized to process upright rather than inverted faces (Peterson \& Rhodes, 2003). In the context of recent findings that face-like objects can be misperceived as faces (pareidolia phenomenon) and that this erroneous perception is accompanied by face-related brain activity (Hadjikhani, Kveraga, Naik, \& Ahlfors, 2009), we surmised that we would observe face-related processing using these stimulus sets.

\section{Methods}

Forty-six undergraduate students (mean age, 19.9 years, $\mathrm{SD}=$ 1.1, 32 females) participated for pay. All reported normal or corrected-to-normal visual acuity and normal color vision.
Fourteen participants were assigned to Experiment 3A, and the remaining 32 participants were assigned to either Experiment $3 \mathrm{~B}$ or Experiment $3 \mathrm{C}$ ( $n=16$, respectively). In Experiment $3 \mathrm{~A}$, the stimuli, apparatus, and procedures were the same as those in Experiment 2 except that the portraits were vertically inverted. Under the bending-forward condition (Fig. 1C, left), the bottom side of the portrait moved toward the participants so that it simulated a situation in which the inverted face bowed. Similarly, the bottom side of the portrait moved backward under the bending-backward condition (Fig. 1C, middle).

The stimuli, apparatus, and procedures in Experiments 3B and $3 \mathrm{C}$ were the same as those in Experiment 1 except that the portraits were replaced with non-face meaningless drawings in Experiment 3B (Fig. 1D) and with face-like objects in Experiment 3C (Fig. 1E). In Experiment 3B, we used 48 black meaningless line drawings (Endo, Saiki, Nakao, \& Saito, 2003). In Experiment 3C, we chose 48 portraits of face-like objects downloaded from the Internet. Each contour shape and the face-like object was placed on a white background subtended $18.8 \mathrm{~cm}$ (vertically) and $13.5 \mathrm{~cm}$ (horizontally).

\section{Results and Discussion}

Figures 8, 9 and 10 show the mean ratings for perceived attractiveness under the bending-forward, bending-backward, and standing-still conditions in Experiments 3A (inverted face), 3B (non-face meaningless drawings), and 3C (face-like object), respectively. Separate ANOVAs on the ratings elicited by a tilting motion were conducted for each experiment. The main effects did not reach significance in Experiment $3 \mathrm{~A}, F(2,26)=$ $0.50, p<.61, \eta_{p}{ }^{2}=0.04$, or Experiment $3 \mathrm{~B}, F(2,30)=1.96, p<$ $0.16, \eta_{p}{ }^{2}=0.12$. In contrast, the main effect in Experiment $3 \mathrm{C}$ was significant, $F(2,30)=9.3, p<0.01, \eta_{p}{ }^{2}=0.38$. Multiple comparisons indicated that the ratings under the bendingforward condition were significantly higher than were those under the bending-backward and standing-still conditions, $t s(15)>3.05, p s<0.02$, whereas no significant difference was observed between ratings under the bending-backward and standing-still conditions, $t(15)=1.63, p=0.37$.

The results of Experiments $3 \mathrm{~A}$ and $3 \mathrm{~B}$ indicate that the bowing motion did not improve the ratings of inverted faces and non-face meaningless drawings. In contrast, the bowing motion enhanced the attractiveness of the face-like objects (Experiment 3C). These results suggest that the effect of bowing was specific to objects perceived as faces.

\section{Experiment 4}

The present experiments indicated that the mere tilting motion of portraits simulating bowing enhanced perceived attractiveness. The purpose of the Experiment 4 was twofold. First, we 
Experiment $3 \mathrm{~A}$

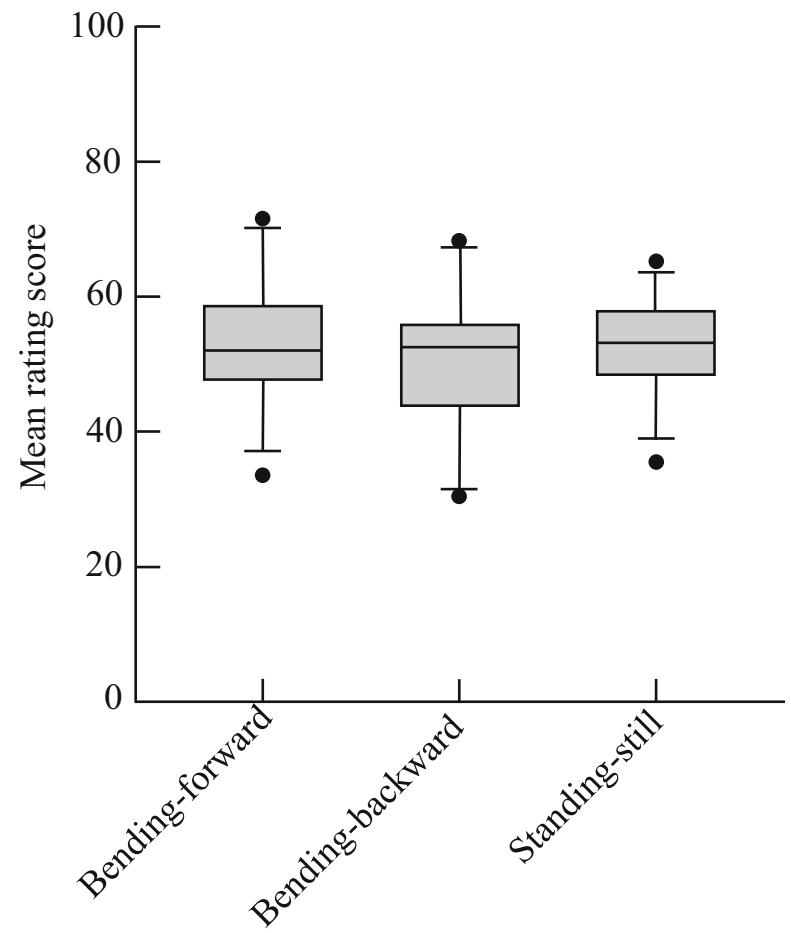

the rotation axis

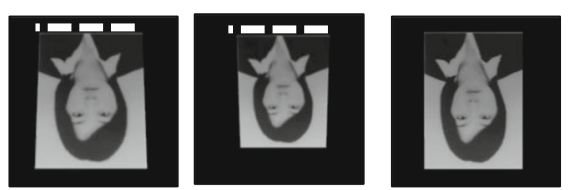

Fig. 8 Box plot for subjective attractiveness under the bending-forward, bending-backward, and standing-still conditions in Experiment 3A. Each box shows the interquartile range, the horizontal line within the box shows the median, and the lines extending from the box to the highest and lowest values, excluding outliers. Outliers are marked separately. The segmented lines in the pictures indicate the rotation axis

replicated the effect of bowing using a different group of participants. Second, and more important, we examined whether bowing increased facial attractiveness when an observer bowed in response to the bowing portraits. We introduced the observers' bowing behavior to examine another alternative explanation: that the effect of bowing observed in the previous experiments was caused by a relative elevation in social status as a side effect of the lack of reciprocity. Specifically, in Asian (e.g., Japanese) cultures, when one person (the sender) bows, the recipient usually bows in return. Such a reciprocal exchange is more naturalistic than is the case in which the recipient does not respond; the exception to this reciprocity involves situations in which the sender's social status is relatively low in relation to that of the recipient. This is because, in Asian cultures, one's status in relation to another person may be reflected by the length and depth of a bow. For example, people who hold lower status usually bow lower and longer. However, people who hold higher status can dispense with
Experiment 3B

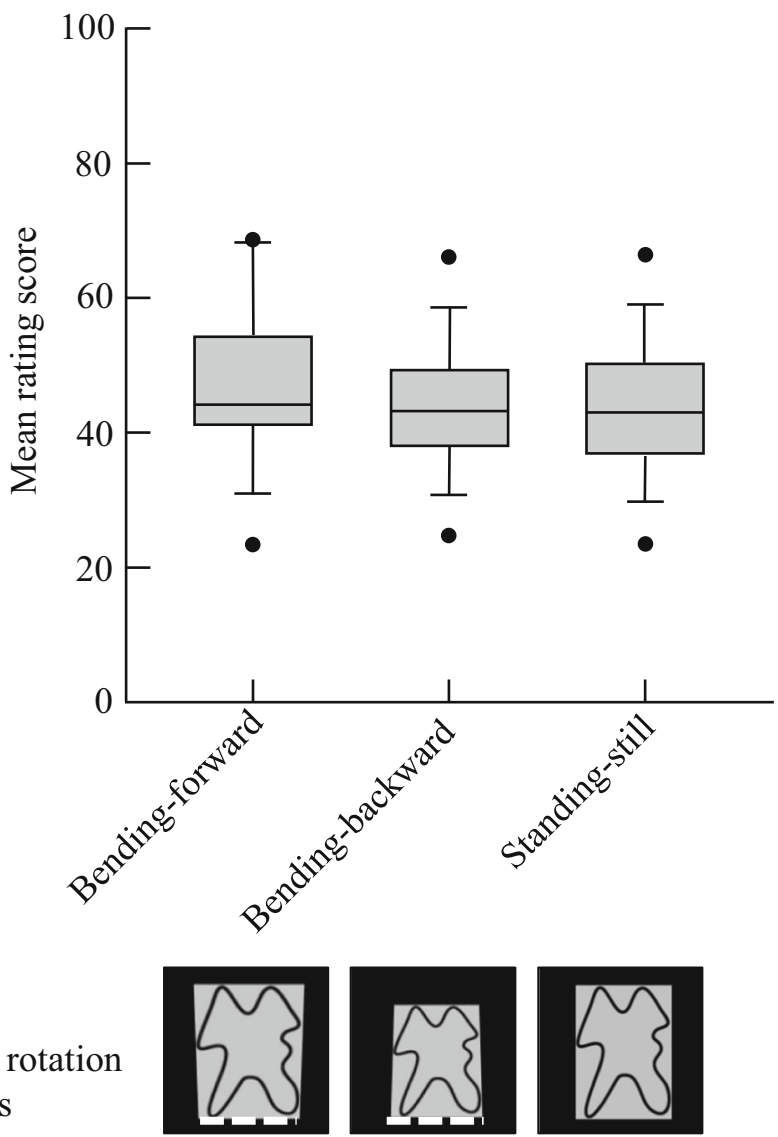

Fig. 9 Box plot for subjective attractiveness under the bending-forward, bending-backward, and standing-still conditions in Experiment 3B. Each box shows the interquartile range, the horizontal line within the box shows the median, and the lines extending from the box to the highest and lowest values, excluding outliers. Outliers are marked separately. The segmented lines in the pictures indicate the rotation axis

bowing; thus, sometimes those people omit bowing and simply nod. Therefore, the participants in Experiments 1 (and 3C) might have misattributed the present circumstance as ones in which they were exempt from bowing due to their elevated social status relative to the bowing figures. Although it is unlikely that such a misattribution toward pictures on a computer screen would arise at a conscious level, we cannot exclude the possibility that such misattribution occurred at an unconscious level. If this were the case, bowing may increase facial attractiveness only when observers omit reciprocal bowing, which may be interpreted as an elevation of the social status of the receiver relative to that of the sender (i.e., the bowing figure). Therefore, in the present experiment, we instructed observers to bow in response to the bowing portraits. Such an in-return bowing should eliminate any misattribution; thus, we predicted a significant reduction in the bowing effect reflected in attractiveness ratings. 


\section{Experiment 3C}

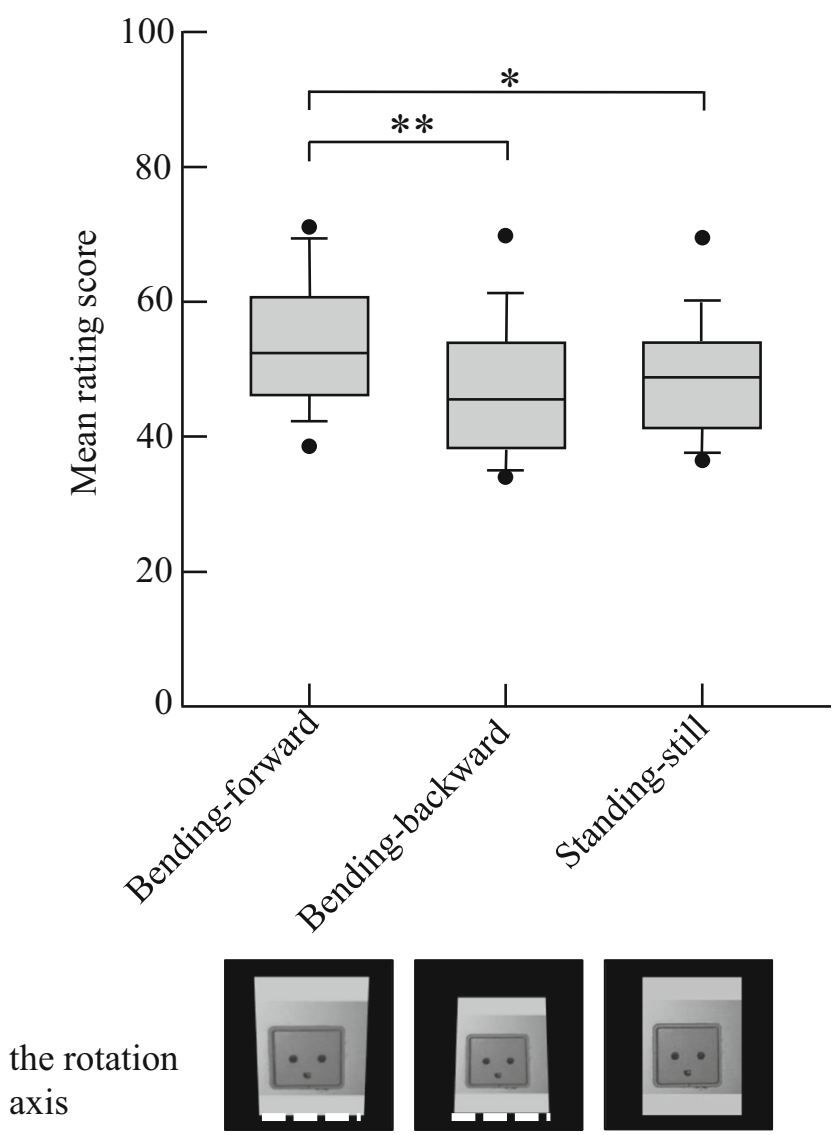

Fig. 10 Box plot for subjective attractiveness under the sliding-forward, sliding-backward, and standing-still conditions in Experiment 3C. Each box shows the interquartile range, the horizontal line within the box shows the median, and the lines extending from the box to the highest and lowest values, excluding outliers. Outliers are marked separately. Black asterisks connected with the solid lines indicate significant differences between conditions $\left({ }^{* *} p<0.01,{ }^{*} p<0.05\right)$. The segmented lines in the pictures indicate the rotation axis

\section{Methods}

Another sample of 16 undergraduate students (mean age, 20.1 years, $\mathrm{SD}=1.6 ; 11$ females) participated for pay. The stimuli, apparatus, and procedures were the same as those in Experiment 1, except that participants were instructed to bow in response to the stimulus display after the figure in the display bowed. The in-return bowing was signaled by a $440-\mathrm{Hz}$ tone that lasted for $100 \mathrm{~ms}$ and began immediately following the bowing of the figure.

\section{Results and Discussion}

The present result replicated the pattern of results from Experiment 1 (Fig. 11). An ANOVA identified a significant main effect of the tilting motion, $F(2,30)=13.27, p<0.01$, $\eta_{p}{ }^{2}=0.47$. Multiple comparisons indicated that the ratings
Experiment 4

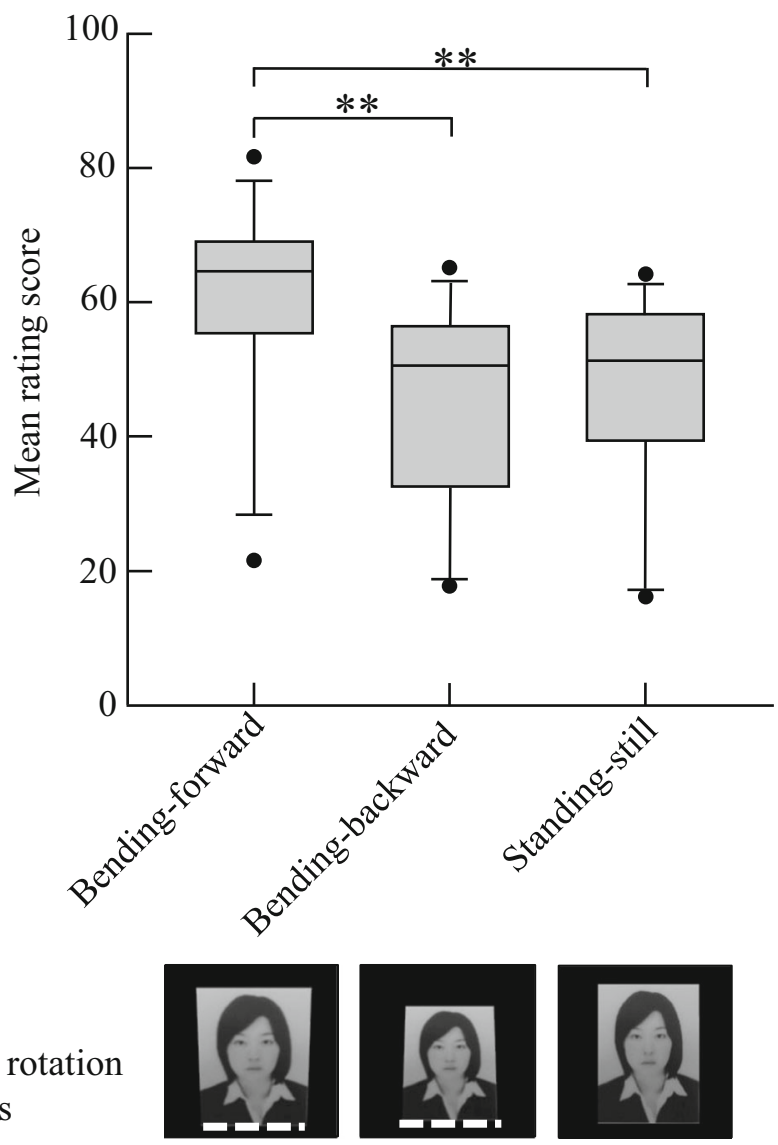

Fig. 11 Box plot for subjective attractiveness under the bending-forward, bending-backward, and standing-still conditions in Experiment 4. Each box shows the interquartile range, the horizontal line within the box shows the median, and the lines extending from the box to the highest and lowest values, excluding outliers. Outliers are marked separately. Black asterisks connected with the solid lines indicate significant differences between conditions $\left(* * p<0.01,{ }^{*} p<0.05\right)$. The segmented lines in pictures indicate the rotation axis

under the bending-forward condition were significantly higher than were those under the bending-backward and the standing-still conditions, $t s(15)>3.62, p \mathrm{~s}<0.01$, whereas no significant difference was observed between the ratings under the bending-backward and the standing-still conditions, $t(15)=0.93, p>0.99$. These results indicate that bowing increased perceived attractiveness.

To test whether the observer's in-return bowing reduced the bowing effect, the magnitude of the bowing effects across Experiments 1 and 4 were compared. We conducted a mixedmodel ANOVA, including one within-subject factor, stimulustilting motion (bending-forward, bending-backward, and standing-still conditions), and one between-subjects factor, observer's in-return bowing (absent in Experiment 1 and present in Experiment 4). The results revealed that the main effect of the tilting motion was significant, $F(2,64)=29.15, p<0.01$, $\eta_{p}{ }^{2}=0.48$. However, the main effect of in-return bowing, $F(1$, 
$32)=0.07, p=0.79, \eta_{p}{ }^{2}<0.01$, and the interaction, $F(2,64)=$ $0.63, p=0.53, \eta_{p}{ }^{2}=0.02$, were not significant. These results suggest that observers' in-return bowing did not reduce the bowing effect, indicating that the effect cannot be attributed to the relative enhancement of social standing of the observers who did not bow during the original experiment.

We next examined the effect of the sex of the observers on the attractiveness rating scores. We combined the data gathered in Experiments 1 and 4 and compared the magnitudes of the bowing effect among male and female observers. A mixed-model ANOVA including one within-subject factor, stimulus-tilting motion (bending-forward, bending-backward, and standing-still conditions), and one between-subjects factor, observer's sex (male and female), revealed a significant main effect of tilting motion, $F(2,64)=27.40, p<0.01, \eta_{p}{ }^{2}=$ 0.46 . However, the main effect of observer's sex, $F(1,32)<$ $0.01, p=0.97, \eta_{p}{ }^{2}<0.01$, and the interaction, $F(2,64)=0.42$, $p=0.66, \eta_{p}{ }^{2}=0.01$, were not significant. These results suggest that the magnitude of the bowing effect did not depend on observers' sex.

\section{Experiment 5}

The experiments conducted thus far consistently have demonstrated that the mere tilting motion of portraits simulating bowing enhanced perceived attractiveness. Why does the bowing motion have positive effects on the perception of facial attractiveness? Experiment 5 examined two alternative explanations. The first is that the bowing motion activates a cognitive schema related to politeness. More specifically, a bowing motion expresses gratitude or respect in Asian cultures and often is associated with verbal expressions of gratitude or apology (Ohashi, 2010). Therefore, a bowing person may be perceived as a polite person with social skills involving the ability to behave appropriately and not emotionally disturb others. Another explanation is that the bowing motion activates a schema related to submissiveness. Previous studies have demonstrated that the head orientation of a target face is an important determinant of perceived relative dominance and submissiveness (Kappas et al., 1994; Mignault \& Chaudhuri, 2003; Rule, Adams, Ambady, \& Freeman, 2012). Furthermore, cross-cultural studies have shown that individuals in East Asian societies perceive stimulus faces signaling submissiveness as more attractive than those signaling dominance (Nakane, 1970; Rosenberger, 1995; Freeman, Rule, Adams, \& Ambady, 2009). It is likely that appeasing behaviors tend to be positively reinforced, because the Japanese culture encourages individuals to be agreeable rather than dominant (Moskowitz et al., 1994; Realo et al., 1997). Given that bowing is one of the most prevalent and important behavioral representations of politeness, submission, and respect, bowing should be positively regarded.
Therefore, Experiment 5 examined whether a bowing motion activated a cognitive schema related to politeness and submissiveness by comparing the scores for facial attractiveness, politeness, and submissiveness under the bending-forward, bending-backward, and standing-still conditions. In this experiment, we used three types of stimuli to demonstrate that bowing affects facial expressions whether the stimuli are female faces (Experiment 5A), male faces (Experiment 5B), or face-like objects (Experiment 5C). Because polite and submissive attitudes are positively regarded in East Asian societies, a similar pattern of results would be expected across these stimulus sets.

Methods

Forty-eight undergraduate students (mean age, 19.5 years, SD $=0.8,32$ females $)$ participated $(16,17$, and 15 participants in Experiments 5A, 5B, and 5C, respectively). All reported normal or corrected-to-normal visual acuity and normal color vision. In Experiment 5A, the stimuli, apparatus, and procedures were the same as those in Experiment 1 except that participants evaluated the politeness and submissiveness of the person after they evaluated attractiveness by clicking a point on a visual analog scale ranging from 0 (impolite or dominant) to 100 (polite or submissive) that appeared under the portrait. The participants were instructed to rate the attractiveness, politeness, and submissiveness of the person according to their own criteria.

The stimuli, apparatus, and procedures in Experiments 5B and $5 \mathrm{C}$ were the same as those in Experiment $5 \mathrm{~A}$ except that the portraits were replaced with portraits of males (Fig. 1) or of the face-like objects used in Experiment 3C (Fig. 1E). In Experiment 5B, we used a 48-stimulus set chosen from 1, 986 male portraits; these were presented on a monitor placed on a table so that the bottom of the monitor was $170 \mathrm{~cm}$ above the floor. This set of portraits was drawn from the middlerange attractiveness group (median 41.4; range 41.2-41.6; rated by 39 female participants who did not participate in the present experiments).

\section{Results and Discussion}

Experiments $5 \mathrm{~A}$ (attractiveness, politeness, and submissiveness) Figure 12 shows the mean ratings for perceived attractiveness, politeness, and submissiveness under the bending-forward, bending-backward, and standing-still conditions. Separate ANOVAs on the ratings elicited by a tilting motion revealed significant main effects for attractiveness, $F(2,30)=18.7, p<0.01, \eta_{p}{ }^{2}=0.55$, politeness, $F(2,30)$ $=14.8, p<0.01, \eta_{p}{ }^{2}=0.50$, and submissiveness, $F(2,30)=$ $15.6, p<0.01, \eta_{p}{ }^{2}=0.51$. Multiple comparisons of the attractiveness scores indicated that the ratings under the bendingforward condition were significantly higher than those under 
Experiment 5A

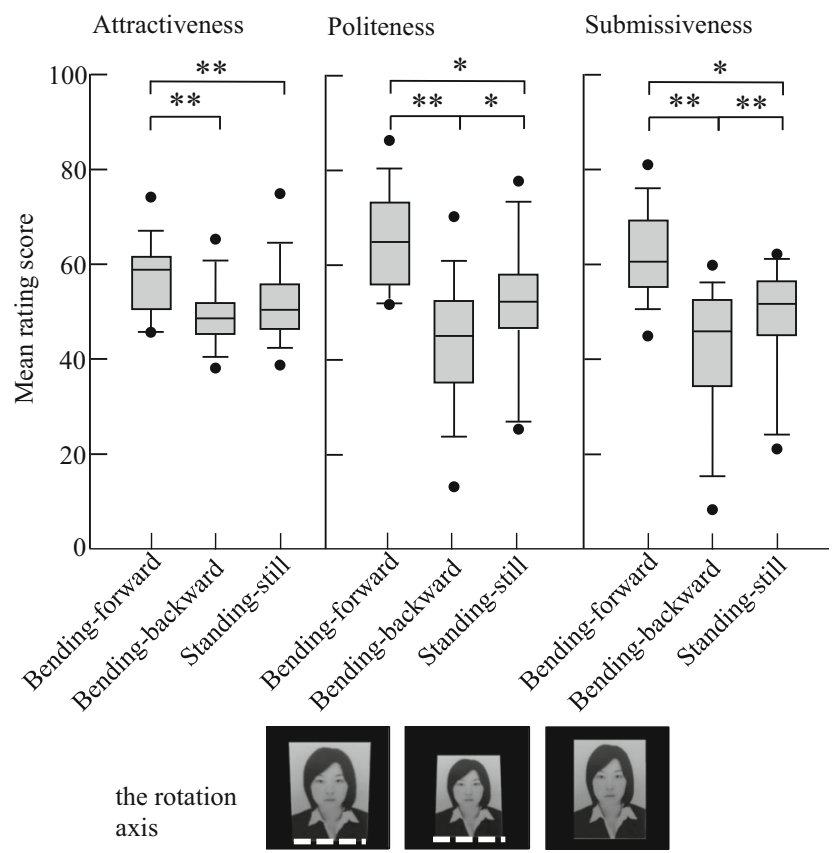

Fig. 12 Box plot for subjective attractiveness, politeness, and submissiveness under the bending-forward, bending-backward, and standing-still conditions in Experiment 5A. Each box shows the interquartile range, the horizontal line within the box shows the median, and the lines extending from the box to the highest and lowest values, excluding outliers. Outliers are marked separately. Black asterisks connected with the solid lines indicate significant differences between conditions $(* * p<0.01, * p<0.05)$. The segmented lines in pictures indicate the rotation axis

the bending-backward and standing-still conditions, $t s(15)>$ $4.61, p \mathrm{~s}<0.01$, whereas no significant differences were observed between ratings under the bending-backward and standing-still conditions, $t(15)=2.58, p=0.06$. The same analyses revealed that ratings under the standing-still condition were significantly lower than those under the bendingforward condition and significantly higher than those under the bending-backward conditions for politeness, $t s(15)>3.11$, $p \mathrm{~s}<0.03$, and submissiveness, $t s(15)>3.22, p \mathrm{~s}<0.02$.

Experiment $5 B$ (male faces) Figure 13 shows the mean ratings for perceived attractiveness, politeness, and submissiveness under the bending-forward, bending-backward, and standing-still conditions. The same analyses revealed significant main effects of ratings of attractiveness, $F(2,32)=42.1, p$ $<0.01, \eta_{p}{ }^{2}=0.72$, politeness, $F(2,32)=66.1, p<0.01, \eta_{p}{ }^{2}=$ 0.81 , and submissiveness, $F(2,32)=67.1, p<0.01, \eta_{p}{ }^{2}=$ 0.81 . Multiple comparisons on the main effects of attractiveness, politeness, and submissiveness indicated that the ratings under the standing-still condition were significantly lower than those under the bending-forward condition and significantly higher than those under the bending-backward conditions for all dependent measures (attractiveness, $t s(16)>4.29$,
Experiment 5B

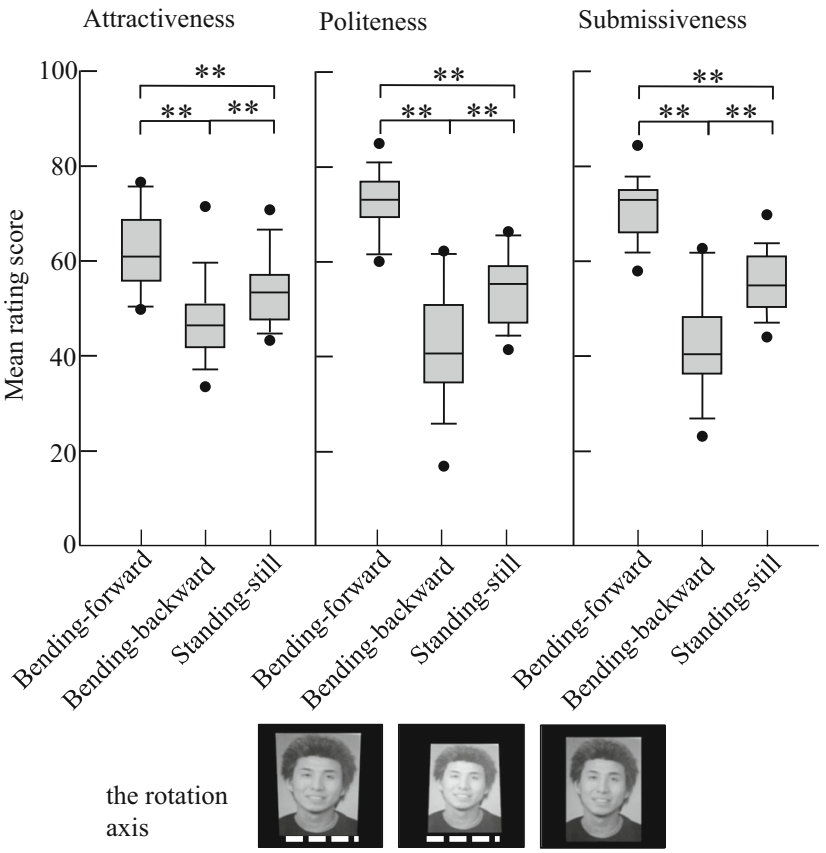

Fig. 13 Box plot for subjective attractiveness, politeness, and submissiveness under the bending-forward, bending-backward, and standing-still conditions in Experiment 5B. Each box shows the interquartile range, the horizontal line within the box shows the median, and the lines extending from the box to the highest and lowest values, excluding outliers. Outliers are marked separately. Black asterisks connected with the solid lines indicate significant differences between conditions $(* * p<$ $0.01, * p<0.05)$. The segmented lines in the pictures indicate the rotation axis

$p \mathrm{~s}<0.01$, politeness, $t s(16)>5.21, p \mathrm{~s}<0.01$, and submissiveness, $t s(16)>6.39, p s<0.01)$.

Examine the differences in the pattern of results between judgments of female versus male faces, we conducted an additional ANOVA on the attractiveness ratings with the motion condition (bending toward, bending backward, or standing still) as a within-subjects factor and stimulus sex (male or female) as a between-subject factor. This analysis identified the significant interaction, $F(2,62)=5, p<0.01, \eta_{p}{ }^{2}=0.14$, as well as a significant main effect of motion condition, $F(2,62)$ $=58.28, p<0.01, \eta_{p}{ }^{2}=0.65$. A simple main effect of stimulus sex under the bending toward condition was marginally significant, $F(1,93)=3.18, p=0.08, \eta_{p}{ }^{2}=0.03$, indicating that the effect of bowing effect tended to be greater for male faces relative to female faces. No other effects were significant. Similar comparisons for politeness and submissive judgments revealed no such interactions.

Experiment 5C (face-like objects) Figure 14 shows the mean ratings of perceived attractiveness, politeness, and submissiveness under the bending-forward, bending-backward, and standing-still conditions. The same analyses conducted above revealed significant main effects for attractiveness, $F(2,28)=$ 


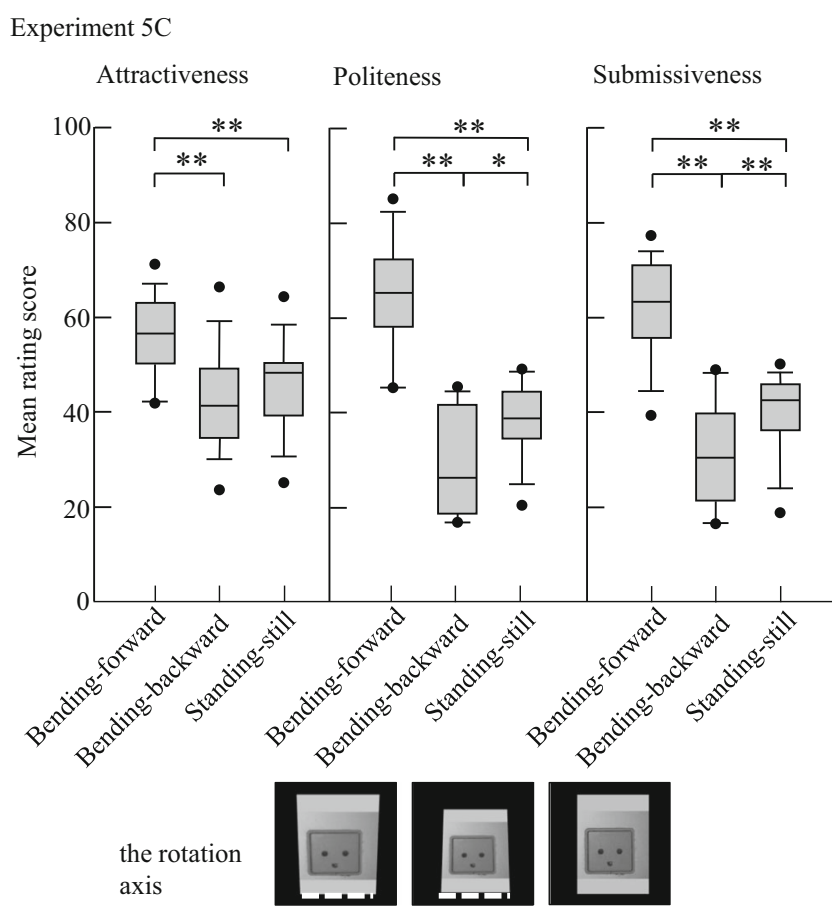

Fig. 14 Box plot for subjective attractiveness, politeness, and submissiveness under the bending-forward, bending-backward, and standing-still conditions in Experiment 5C. Each box shows the interquartile range, horizontal line within the box shows the median, and the lines extending from the box to the highest and lowest values, excluding outliers. Outliers are marked separately. Black asterisks connected with the solid lines indicate significant differences between conditions $(* * p<$ $\left.0.01,{ }^{*} p<0.05\right)$. The segmented lines in the pictures indicate the rotation axis

18.7, $p<0.01, \eta_{p}{ }^{2}=0.57$, politeness, $F(2,28)=44.8, p<0.01$, $\eta_{p}{ }^{2}=0.76$, and submissiveness, $F(2,28)=44.8, p<0.01, \eta_{p}{ }^{2}$ $=0.76$. In terms of attractiveness, multiple comparisons indicated that the ratings under the bending-forward condition were significantly higher than those under the bendingbackward and standing-still conditions, ts(14) $>4.59, p$ s $<$ 0.01 , whereas no significant differences were found between ratings under the bending-backward and standing-still conditions, $t(14)=1.74, p=0.31$. The same analyses indicated that ratings under the standing-still condition were significantly lower than those under the bending-forward condition and significantly higher than those under the bending-backward condition for politeness, $t s(14)>3.48, p \mathrm{~s}<0.02$, and submissiveness, $t s(14)>3.82, p \mathrm{~s}<0.01$.

The results of Experiments 5A-C indicated that the bowing motion improved the attractiveness, politeness, and submissiveness ratings of female and male faces and face-like objects. These results show that the effect of bowing was consistent and robust across a variety of stimulus sets perceived as depicting faces. Furthermore, the present results revealed that the bowing motion increased not only perceived attractiveness but also perceived politeness and submissiveness. This finding suggests that the perceived attractiveness of faces was enhanced, because the cognitive schemas of politeness and submissiveness were activated. Furthermore, the results indicated that the bending-backward motion decreased the ratings of politeness and submissiveness but not of attractiveness, except for the male faces. These findings may indicate that impolite and dominant behavior (i.e., the bending-backward motion) does not affect facial attractiveness relative to polite and submissive behavior (i.e., the bending-forward motion). One possible explanation of this finding is that the bendingbackground motion may be perceived as an increase in the relative distance, and thus, the unpleasantness of the face may be reduced. However, this motion may have decreased the attractiveness ratings of male portraits in particular.

\section{Experiment 6}

The results of Experiments 1 through 5 consistently demonstrated that the perception of the tilting motion of facial portraits as bowing was critical to the enhancement of their perceived attractiveness. However, one could argue that tilting flat portraits would yield results that differ from those with ordinary three-dimensional faces. Thus, it remained unclear whether tilting the portraits would elicit the same effect as tilting 3D faces. To examine whether the bowing motion of 3D faces elicited equivalent enhancement in perception of attractiveness, we used movie clips of 48 computergenerated female faces and measured the attractiveness ratings of the 3D faces during bowing.

\section{Methods}

Another sample of 16 undergraduate students (mean age, 19.6 years, $\mathrm{SD}=0.5 ; 12$ females) participated for pay. The experimental procedure and rendering of the stimulus display were programmed using Matlab and Psychophysics Toolbox (Brainard, 1997; Pelli, 1997). The viewing distance was approximately $57 \mathrm{~cm}$, and the monitor was placed on a table so that the bottom of the monitor was $90 \mathrm{~cm}$ above the floor.

Stimuli consisted of 48 computer-generated faces (Japanese females) with different clothing and hairstyles. All displayed a neutral expression or a faint smile. Each face was randomly created using Facegen modeler 3.5 (Singular Inversions, Toronto) and was applied to the 3D model selected from the library of Daz studio (Daz 3D, Salt Lake City). Also, the animation was created by using Daz studio. Each picture of 3D face subtended $17.4 \mathrm{~cm}$ in height and $17.4 \mathrm{~cm}$ in width.

Design and Procedure The experiment consisted of familiarization and test periods. During the familiarization period, a stream of the upright 3D faces used in the experimental session was presented successively (duration $=1 \mathrm{~s}$ each) while participants viewed the stream. In the next stage, participants 
rated the faces under three (bending-forward, bending-backward, and standing-still) conditions. The experiment consisted of 144 trials (48 under each condition, mixed randomly within a block.) Each identity (facial stimulus) appeared three times, and each was assigned with equal frequency to the three conditions. All animations started with the upright faces gazing forward for 1,000 ms; this was followed by a sequence of smoothly tilting motions under the bending-forward and bending-backward conditions (Fig. 3). During the tiltingmotion sequence, the body of the 3D model was bent to approximately 25 degrees for $500 \mathrm{~ms}$ and then returned to the upright position for the same duration. The bending-backward condition was the same as the bending-forward condition except that the body was bent in the reverse direction. The face remained upright for 2,000 ms under the standing-still condition. Then, participants evaluated the perceived attractiveness of the person by clicking a point on the same visual analog scale used in Experiment 1.

\section{Results and Discussion}

The present result replicated the results of Experiment 1 (Fig. 15). ANOVA identified a significant main effect of the tilting motion, $F(2,30)=17.88, p<0.01, \eta_{p}{ }^{2}=0.54$. Multiple comparisons indicated that the ratings under the bendingforward condition were significantly higher than those under the bending-backward and the standing-still conditions, $t s(15)$ $>4.26, p \mathrm{~s}<0.01$, whereas no significant difference was observed between the ratings under the bending-backward and the standing-still conditions, $t(15)=2.3, p=0.11$. These results indicate that the bowing motion of $3 \mathrm{D}$ faces increased their perceived attractiveness.to a degree equivalent to that found with the 2D stimuli.

\section{General Discussion}

The present study focused on bowing as a greeting behavior and examined its modulatory effect on perception of attractiveness. The results of Experiment 1 indicate that the mean attractiveness rating of the bowing portrait was significantly higher than was that of the bending-backward and standingstill portraits. Additional control experiments revealed that alternative accounts relying on apparent spatial proximity and physical characteristics, such as femininity and relative social standing, could not solely explain the effect of bowing and indicated that the effect was specific to objects perceived as faces. Specifically, sliding the bottom side of the portrait toward or away from participants (Experiment 2) and portraits that were static but tilted toward or away from participants (Experiment 2A) did not increase attractiveness ratings. No differences in ratings were found among the static (bendingforward bending-backward, and upright) images. Critically, the instruction to note that the portrait was merely tilted
Experiment 6

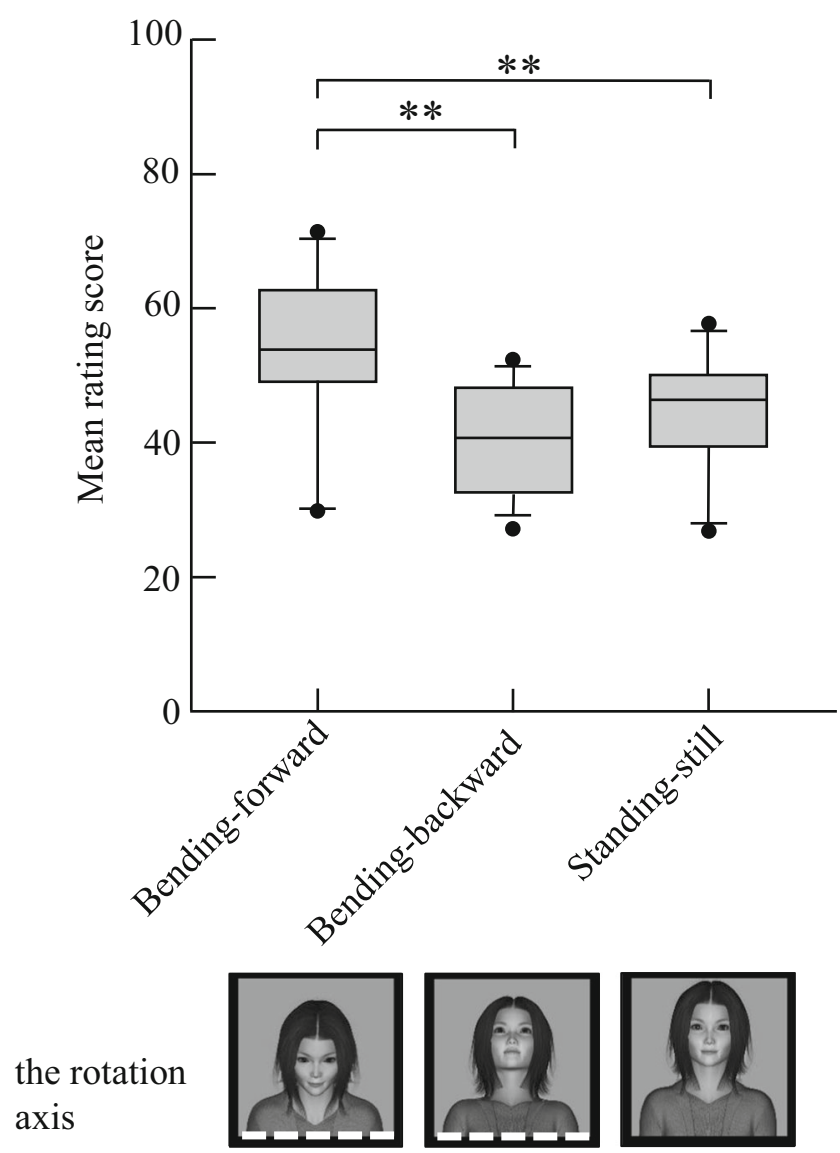

Fig. 15 Box plot for subjective attractiveness under the sliding-forward, sliding-backward, and standing-still conditions in Experiment 6. Each box shows the interquartile range, horizontal line within the box shows the median, and the lines extending from the box to the highest and lowest values, excluding outliers. Outliers are marked separately. Black asterisks connected with the solid lines indicate significant differences between conditions $(* * p<0.01, * p<0.05)$. The segmented lines in the pictures indicate the rotation axis

forward or backward eliminated the enhancement effect of bowing even when the motion stimuli used were identical to those in Experiment 1. The bowing motion did not enhance the ratings of inverted faces or non-face meaningless drawings (Experiments 3A and 3B). The bowing motion did enhance the attractiveness of face-like objects (Experiment 3C). Inreturn bowing behavior by observers did not reduce the bowing effect, suggesting that the effect cannot be attributed to the misinterpretation of the social standing of the observers, who had merely received and did not exchange bows in the original experiment (Experiment 4). Furthermore, the bowing motion likely activated cognitive schemas related to politeness and submissiveness (Experiment 5). Finally, movie clips of realistic 3D faces elicited the same effect as the tilting motion of still portraits did (Experiment 6). These results suggest that the tilting motion of facial portraits (and face-like objects), which 
mimics bowing, enhanced perceived attractiveness, at least in a culture familiar with greeting by bowing.

The human perceptual system combines information regarding physical beauty and social cues, such as shifts in gaze (Mason et al., 2005; Kampe, et al., 2001; Conway, et al., 2008), facial expressions (Reis et al., 1990), and head orientation (Kampe et al., 2001) to maximize the potential benefits of the encounter. Recent studies have extended this notion by demonstrating that the human perceptual system also integrates the vertical head angle when determining facial impressions (Mignault \& Chauderi, 2003; Burke \& Sulikowski, 2010). However, these studies did not manipulate the vertical motion of facial images. Thus, there is little evidence that the bowing motion that serves as a greeting behavior affects perceived attractiveness. The present study addressed this issue, and the results indicate that the mere tilting motion of portraits simulating bowing enhanced perceived attractiveness, suggesting that the human perceptual system combines information regarding the bowing motion with information regarding physical characteristics, such as beauty, politeness, goodness, and hospitality. This is the first study to measure systematically the effect of bowing on perception of attractiveness.

In the context of previous studies demonstrating that a vertical face orientation influences perceived impression (Burke \& Sulikowski, 2010; Mignault \& Chauderi, 2003), the present results demonstrate that the mere tilting motion of portraits affect perception of facial attractiveness. These findings indicate that the human visual system evaluates facial impressions by integrating the perceived pitch angle. A new finding was that the tilting motion of facial portraits markedly increased the subjective attractiveness of faces as previous studies used static images, leaving the effect of the bowing motion on perception of attractiveness unclear. This effect was not due to the mere physical characteristics of static images. The present results suggest that the bowing motion is a necessary condition for enhancing the perceived attractiveness of portraits viewed under the present stimulus parameters.

Although apparent spatial proximity between the stimulus and observer could not, by itself, explain the effect of bowing, it is possible that bowing is one of the social cues that provide information regarding motivation to approach. Previous studies have demonstrated that reduced apparent spatial distance (or motivation latency to shorten the relative distance) increases the positive rating of target objects (Cacioppo et al., 1993; Markman \& Brendl, 2005). Furthermore, social cues, such as the gaze direction, facial expression, and bodily gestures of the stimulus, convey signals relevant to whether observers should approach or avoid the stimulus (Adams \& Kleck, 2003, 2005; Willis et al., 2011; Van Peer, Rotteveel, Spinhoven, Tollenaar, \& Roelofs, 2010). Such social cues are likely to trigger a variety of processes that may shape the person's interpretation of the situation; thus, identification of such information helps to maximize the potential benefits of the encounter by helping an observer select the most attractive individual with a social interest in her or him (Mason et al., 2005; Jones et al., 2006). The present results extend the notion that social cues can be integrated into perceptions of attractiveness by demonstrating that bowing increased facial attractiveness even when such task-irrelevant primitive visual motion was presented in conjunction with target facial images. We surmise that bowing is a social cue that implies approach motivation.

The present results reveal that perceiving the objects in the images as faces was critical to enhance the perception of attractiveness when the tilting motion of the images was viewed. Several studies have shown that inverted faces and face-like objects are useful for investigation of the processing of facial stimuli and social interactions involving faces. More specifically, previous studies have demonstrated that inverted presentation impairs facial processing (, Yin, 1969; Valentine, 1988); thus, the effect of a social cue such as a direct gaze does not enhance the perception of attractiveness (Ewing et al., 2010). In contrast, face-like objects, such as a picture of electric outlet, can be misperceived as faces (Hadjikhani et al., 2009), and such misperception of an object in an image is important for eliciting social attention (Takahashi \& Watanabe, 2013). These findings suggest that the ability of social cues to affect perceptual processing (e.g., related to attractiveness) and attentional orienting depends on whether it is a face that is perceived. The present findings extend this idea by demonstrating that the effect of a bowing motion is specific to face (and face-like) stimuli. The tilting motion is probably perceived as greeting behavior within the social context of simulating greeting.

The present findings demonstrate that a bowing motion activates cognitive schemas related to politeness and submissiveness. It has been shown that a bowing motion expresses gratitude or respect in Asian cultures (Ohashi, 2010) and that a lower head orientation is related to an impression of the face as submissive (Kappas et al., 1994; Mignault \& Chaudhuri, 2003; Rule, et al., 2012). Furthermore, because the Japanese culture typically encourages individuals to be agreeable rather than dominant (Moskowitz et al., 1994; Realo et al., 1997), behaving in a polite and submissive manner would be expected to result in one's being perceived as more attractive (Nakane, 1970; Rosenberger, 1995; Freeman, et al., 2009). Therefore, the bowing motion of the portraits would be expected to activate associations with politeness and submissiveness, which would then be integrated into evaluations of faces' attractiveness.

Notably, we examined the effect of bowing on the perception of attractiveness in a culture familiar with greeting by bowing. What is clear from the present findings is that bowing faces, regardless of the types (real Japanese faces, softwaredefined Asian faces, or artificial objects), are perceived attractive at least for Japanese viewers. We must be careful with 
respect to generalizing this finding because the tilting motion of the portrait may be differently perceived or interpreted in different cultures. There is much evidence regarding crosscultural variations in the interpretation of social signals (e.g., Elfenbein \& Ambady, 2002; Akechi, Senju, Uibo, Kikuchi, Hasegawa, \& Hietanen, 2013). For example, East Asians tend to interpret faces with a fearful expression as surprised and those with an expression of disgust as angry (e.g., Elfenbein \& Ambady, 2002). Moreover, when a gaze is directed toward an observer, individuals from an East Asian culture perceive the gazer's face as more unapproachable and unpleasant than do individuals from a Western culture (Akechi et al., 2013). That is, individuals from East Asian and Western cultures may interpret the same social cue differently according to their respective cultural norms. In an extreme case, the tilting motion of a portrait may be perceived as a person falling forward in a culture unfamiliar with greeting by bowing. Further study is needed to clarify the relationship between cultural background and the bowing motion.

In conclusion, the present results demonstrate that a tilting motion of portraits (or images of face-like objects) that mimics bowing enhances perceived attractiveness. Furthermore, additional control experiments revealed that alternative accounts relying on apparent spatial proximity, physical characteristics, and social standing could not solely explain the effect of bowing, indicating that the effect was specific to objects perceived as faces. These results suggest that the visual system may combine information regarding bowing as a greeting behavior with information regarding physical beauty at least in a culture familiar with greeting by bowing.

Acknowledgments This work was supported by Grant-in-Aid for Scientific Research on Innovative Areas, "Face perception and recognition" from MEXT KAKENHI 23119731 and 26590183 to JK. We thank Shuto Chi-na for collecting data.

\section{References}

Adams, R. B., Jr., \& Kleck, R. E. (2003). Perceived gaze direction and the processing of facial displays of emotion. Psychological Science, 14, 644-647.

Adams, R. B., Jr., \& Kleck, R. E. (2005). The effects of direct and averted gaze on the perception of facially communicated emotion. Emotion, 5, 3-11.

Akechi, H., Senju, A., Uibo, H., Kikuchi, Y., Hasegawa, T., \& Hietanen, J. K. (2013). Attention to eye contact in the West and East: Autonomic responses and evaluative ratings. PLoS ONE, 8, e59312.

Andersson, M. (1994). Sexual selection. Princeton: NJ7 Princeton Univ. Press.

Argyle, M., \& Cook, M. (1976). Gaze and mutual gaze. Cambridge, England: Cambridge University Press.

Baron-Cohen, S. (1995). Mindblindness: An essay on autism and theory of mind. Cambridge, MA: MIT Press.

Brainard, D. H. (1997). The psychophysics toolbox. Spatial Vision, 10, 433-436
Burke, D., \& Sulikowski, D. (2010). A new viewpoint on the evolution of sexually dimorphic human faces. Evolutionary Psychology, 8, 573585 .

Buss, D. M., \& Barnes, M. (1986). Preferences in human mate selection. Journal of Personality and Social Psychology, 50, 559-570.

Cacioppo, J. T., Priester, J. R., \& Bernston, G. G. (1993). Rudimentary determinants of attitudes. II: Arm flexion and extension have differential effects on attitudes. Journal of Personality and Social Psychology, 65, 5-17.

Cash, T. F., \& Kilcullen, R. N. (1985). The eye of the beholder: Susceptibility to sexism and beautyism in the evaluation of managerial applicants. Journal of Applied Social Psychology ,15, 591605.

Chaplin, W. F., Phillips, J. B., Brown, J. D., Clanton, N. R., \& Stein, J. L. (2000). Handshaking, gender, personality, and first impressions. Journal of Personality and Social Psychology, 79, 110-117.

Conway, C. A., Jones, B. C., DeBruine, L. M., \& Little, A. C. (2008). Evidence for adaptive design in human gaze preference. Proceedings of the Royal Society of London, Series B: Biological Sciences, 275, 63-69.

Elfenbein, H. A., \& Ambady, N. (2002). On the universality and cultural specificity of emotion recognition: A meta-analysis. Psychological Bulletin, 128, 203-235.

Emery, N. J. (2000). The eyes have it: The neuroethology, function and evolution of social gaze. Neuroscience and Biobehavioral Reviews, 24, 581-604.

Endo, N., Saiki, J., Nakao, Y., \& Saito, H. (2003). Perceptual judgments of novel contour shapes and hierarchical descriptions of geometrical properties. Japanese Journal of Psychology, 74, 346-353.

Ewing, L., Rhodes, G., \& Pellicano, E. (2010). Have you got the look? Gaze direction affects facial attractiveness. Visual Cognition, 18, $321-330$

Freeman, J. B., Rule, N. O., Adams, R. B., Jr., \& Ambady, N. (2009). Culture shapes a mesolimbic response to signals of dominance and subordination that associates with behavior. NeuroImage, 47, 353359.

Freyd, J. J., \& Finke, R. A. (1984). Representational momentum. Journal of Experimental Psychology: Learning, Memory, and Cognition, 10, $126-132$.

Hadjikhani, N., Kveraga, K., Naik, P., \& Ahlfors, S. P. (2009). Early (M170) activation of face-specific cortex by face-like objects. Neuroreport, 20, 403-407.

Jones, B. C., DeBruine, L. M., Little, A. C., Conway, C. A., \& Feinberg, D. R. (2006). Integrating gaze direction and expression in preferences for attractive faces. Psychological Science, 17, 588-591.

Kampe, K. K. W., Frith, C. D., Dolan, R. J., \& Frith, U. (2001). Reward value of attractiveness and gaze. Nature, 413, 589.

Kappas, A., Hess, U., Barr, C., \& Kleck, R. E. (1994). Angles of regard: The effect of vertical viewing angle on the perception of facial expressions. Journal of Nonverbal Behavior, 18, 263-280.

Langlois, J. H., \& Roggman, L. A. (1990). Attractive faces are only average. Psychological Science, 1, 115-121.

Markman, A. B., \& Brendl, M. (2005). Constraining theories of embodied cognition. Psychological Science, 16, 6-10.

Mason, M. F., Tatkow, E. P., \& Macrae, C. N. (2005). The look of love: Gaze shifts and person perception. Psychological Science, 16, 236239.

Mignault, A., \& Chaudhuri, A. (2003). The many faces of a neutral face: Head tilt and perception of dominance and emotion. Journal of Nonverbal Behavior, 27, 111-132.

Møller, A. P., \& Thornhill, R. (1998). Bilateral symmetry and sexual selection: A meta-analysis. American Naturalist, 151, 174-192.

Moskowitz, D. S., Suh, E. J., \& Desaulniers, J. (1994). Situational influences on gender differences in agency and communion. Journal of Personality and Social Psychology, 66, 753-761.

Nakane, C. (1970). Japanese Society. Middlesex: Penguin Books. 
Ohashi, J. (2010). Balancing obligations: Bowing and linguistic features in thanking in Japanese. Journal of Politeness Research, 6, 183-214.

Pelli, D. G. (1997). The VideoToolbox software for visual psychophysics: Transforming numbers into movies. Spatial Vision, 10, 437-442.

Peterson, M. A., \& Rhodes, G. (Eds.). (2003). Perception of faces, objects and scenes: Analytic and holistic processes. New York: Oxford University Press.

Realo, A., Allik, J., \& Vadi, M. (1997). The hierarchical structure of collectivism. Journal of Research in Personality, 31, 93-116.

Reis, H. T., Wilson, I. M., Monestere, C., Bernstein, S., Clark, K., Seidl, E., Franco, M., Gioioso, E., Freeman, L., \& Radoane, K. (1990). What is smiling is beautiful and good. European Journal of Social Psychology, 20, 259-267.

Rosenberger, N. R. (1995). Japanese Sense of Self. Cambridge: Cambridge University Press.

Rule, N. O., Adams, R. B., Jr., Ambady, N., \& Freeman, J. B. (2012). Perceptions of dominance following glimpses of faces and bodies. Perception, 41, 687-706.

Sato, S., \& Kawahara, J. (2014). Attentional capture by completely taskirrelevant faces. Psychological Research. doi:10.1007/s00426-0140599-8
Sigall, H. \& Ostrove, N. (1975) Beautiful but dangerous: Effects of offender attractiveness and nature of the crime on juridic judgments. Journal of Personality and Social Psychology, 31, 410-414.

Stewart, G. L., Dustin, S. L., Barrick, M. R., \& Darnold, T. C. (2008). Exploring the handshake in employment interviews. Journal of Applied Psychology, 8, 1139-1146.

Takahashi, K., \& Watanabe, K. (2013). Gaze cueing by pareidolia faces. $i$ Perception, 4(8), 490-492.

Valentine, T. (1988). Upside-down faces. A review of the effect of inversion upon face recognition. British Journal of Psychology, 79, 471491.

Van Peer, J. M., Rotteveel, M., Spinhoven, P., Tollenaar, M. S., \& Roelofs, K. (2010). Affect-congruent approach and withdrawal movements of happy and angry faces facilitate affective categorization. Cognition \& Emotion, 24, 863-875.

Willis, M. L., Palermo, R., \& Burke, D. (2011). Judging approachability on the face of it: The influence of face and body expressions on the perception of approachability. Emotion, 11, 514-523.

Yin, R. K. (1969). Looking at upside-down faces. Journal of Experimental Psychology, 81, 141-145. 